\title{
The PML for rough surface scattering
}

\author{
Article
}

Accepted Version

Chandler-Wilde, S. N. and Monk, P. (2009) The PML for rough surface scattering. Applied Numerical Mathematics, 59 (9). pp. 2131-2154. ISSN 0168-9274 doi: https://doi.org/10.1016/j.apnum.2008.12.007 Available at https://centaur.reading.ac.uk/1611/

It is advisable to refer to the publisher's version if you intend to cite from the work. See Guidance on citing.

To link to this article DOI: http://dx.doi.org/10.1016/j.apnum.2008.12.007

Publisher: Elsevier

All outputs in CentAUR are protected by Intellectual Property Rights law, including copyright law. Copyright and IPR is retained by the creators or other copyright holders. Terms and conditions for use of this material are defined in the End User Agreement.

\section{www.reading.ac.uk/centaur}

\section{CentAUR}

Central Archive at the University of Reading

Reading's research outputs online 


\title{
THE PML FOR ROUGH SURFACE SCATTERING
}

\author{
SIMON N. CHANDLER-WILDE $\dagger$ AND PETER MONK $\ddagger$
}

\begin{abstract}
In this paper we investigate the use of the perfectly matched layer (PML) to truncate a time harmonic rough surface scattering problem in the direction away from the scatterer. We prove existence and uniqueness of the solution of the truncated problem as well as an error estimate depending on the thickness and composition of the layer. This global error estimate predicts a linear rate of convergence (under some conditions on the relative size of the real and imaginary parts of the PML function) rather than the usual exponential rate. We then consider scattering by a half-space and show that the solution of the PML truncated problem converges globally at most quadratically (up to logarithmic factors), providing support for our general theory. However we also prove exponential convergence on compact subsets. We continue by proposing an iterative correction method for the PML truncated problem and, using our estimate for the PML approximation, prove convergence of this method. Finally we provide some numerical results in 2D.
\end{abstract}

1. Introduction. In the context of scattering theory, a rough surface is a perturbation, possibly nonlocal, of an infinite plane such that the whole surface lies within a finite distance of the original plane. Such surfaces arise frequently in applications, for example in modeling scattering from the ground or sea.

Rough surface scattering problems are the subject of intensive studies in the engineering literature, with a view to developing both rigorous methods of computation and approximate, asymptotic, or statistical methods (see e.g. the reviews and monographs by Ogilvy [19], Voronovich [22], Saillard \& Sentenac [20], Warnick \& Chew [23], and DeSanto [10]). The standard way of approximating such problems is to use boundary integral techniques. However, variational domain formulations discretised with finite elements are also widely used, especially in the case when the boundary is periodic (e.g. [3, 12]). Moreover, using variational techniques [4], we have been able to extend the existence and uniqueness theory for the sound soft acoustic scattering problem to more general surfaces than was possible using integral equation techniques. In this paper we shall consider the use of a variational technique to approximate the sound soft acoustic problem based on the use of the perfectly matched layer (PML) to truncate the computational domain in the direction away from the scatterer.

We now define some notation, then state the problem we shall study before returning to a further discussion of the contents of the paper for the rest of this introduction. For $n=2,3$ let $x=\left(\tilde{x}, x_{n}\right)$ where $\tilde{x} \in \mathbb{R}^{n-1}$. Let $U_{H}=\left\{x: x_{n}>H\right\}$ with boundary $\Gamma_{H}=\left\{(\tilde{x}, H): \tilde{x} \in \mathbb{R}^{n-1}\right\}$. The domain of the acoustic field is denoted $D \subset \mathbb{R}^{n}$ with boundary $\Gamma=\partial D$. We assume that $D$ is a connected open set such that there are constants $f_{-}$and $f_{+}$with

$$
U_{f_{+}} \subset D \subset U_{f_{-}} .
$$

Thus the boundary $\Gamma$ of $D$ lies between the planes $x_{n}=f_{-}$and $x_{n}=f_{+}$(see Fig. 2.1 for a diagram of the rough surface and notation).

The variational technique we shall use as a basis of this study is formulated by restricting the scattering problem to a strip above $\Gamma$ so, for each $a \geq f_{+}$, we define

$$
S_{a}=D \backslash \bar{U}_{a}
$$

and denote by $\nu$ the unit outward normal to $S_{a}$. Using $S_{a}$ we can define an appropriate solution space. For any $a \geq f_{+}$we define

$$
V_{a}=\left\{\left.\phi\right|_{S_{a}}: \phi \in H_{0}^{1}(D)\right\}
$$

${ }^{*}$ This work was supported by the UK Engineering and Physical Sciences Research Council under Grant GR/S67401, and by Visiting Fellowships of the Isaac Newton Institute for Mathematical Sciences. The research of Peter Monk is also supported by AFOSR grant F49620-02-1-0071.

${ }^{\dagger}$ Department of Mathematics, University of Reading, Whiteknights, PO Box 220 Berkshire, RG6 6AX, UK (S.N.Chandler-Wilde@reading.ac.uk)

${ }_{\ddagger}^{\ddagger}$ Department of Mathematical Sciences, University of Delaware, Newark, DE 19716, USA (monk@math.udel.edu) 
equipped with the parameter dependent inner-product

$$
(u, v)_{V_{a}}:=\int_{S_{a}}\left(\nabla u \cdot \nabla \bar{v}+k^{2} u \bar{v}\right) d x
$$

where $\bar{v}$ denotes the complex conjugate of $v$, and $k>0$ is the wave-number of the acoustic field (see equation (1.3) below). The resulting norm is denoted $\|v\|_{V_{a}}:=\sqrt{(v, v)_{V_{a}}}$.

Now we can state the time harmonic scattering problem we shall study. Given $g \in L^{2}(D)$, with support in $S_{H}$ for some $H \geq f_{+}$, and the wavenumber $k>0$, we wish to approximate the acoustic field $u \in V_{a}$ for every $a \geq f_{+}$that satisfies the Helmholtz equation

$$
\Delta u+k^{2} u=g \quad \text { in } D
$$

in a distributional sense and such that $u$ also satisfies the radiation condition

$$
u(x)=\frac{1}{(2 \pi)^{(n-1) / 2}} \int_{\mathbb{R}^{n-1}} \exp \left(\mathrm{i}\left[\left(x_{n}-H\right) \sqrt{k^{2}-\xi^{2}}+\tilde{x} \cdot \xi\right]\right) \hat{u}_{H}(\xi) d \xi
$$

for $x \in U_{H}$ where $\xi^{2}=\xi \cdot \xi=|\xi|^{2}$, and $\hat{u}_{H}=\left.\mathcal{F} u\right|_{\Gamma_{H}}$ is the Fourier transform of $\left.u\right|_{\Gamma_{H}}$. Note also that $\sqrt{k^{2}-\xi^{2}}=\mathrm{i} \sqrt{\xi^{2}-k^{2}}$ when $|\xi|>k$ and that we normalize the Fourier transform as follows:

$$
(\mathcal{F} \phi)(\xi)=\frac{1}{(2 \pi)^{(n-1) / 2}} \int_{\mathbb{R}^{n-1}} \exp (-\mathrm{i} \tilde{x} \cdot \xi) \phi(\tilde{x}) d \tilde{x}
$$

for $\xi \in \mathbb{R}^{n-1}$. Of course the assumption that $u \in V_{a}$ implies the sound soft boundary condition

$$
u=0 \quad \text { on } \Gamma=\partial D .
$$

In [4] we used a variational formulation, to be described in the next section, and the Lax-Milgram lemma to show that the sound soft scattering problem (1.3), (1.4) and (1.6) has a unique solution in $V_{a}$ without further restriction on $D$ provided

$$
0<k<\sqrt{2} /\left(H-f_{-}\right) .
$$

In the case of general (i.e. larger) $k$ we were able to show the existence of a unique solution by Rellich-type identities coupled with the Babuška-Brezzi theory, under an extra "non-trapping" condition on $D$ that

$$
\text { if } x \in D \text { then } x+s e_{n} \in D \text {, for all } s>0,
$$

where $e_{n}$ denotes the unit vector in the direction $x_{n}$. For simplicity, in this paper we shall allow a general $k>0$ and assume that $D$ satisfies (1.8). However all our results also hold for general $D$ in the low frequency case when (1.7) holds, except that constants depending on the Babuška-Brezzi inf-sup constant for the scattering problem must be modified accordingly.

Our previous variational formulation uses the Dirichlet to Neumman map on $\Gamma_{H}$ to provide a boundary condition there. As part of a numerical scheme this would require the fast calculation of an appropriate singular integral operator. An alternative approach, which avoids the singular integral operator, is to terminate the computational domain using the PML. This paper is devoted to setting up and performing an analysis of such a method motivated by the work of Chen and Wu [7].

We shall only consider the sound soft rough surface scattering problem in this paper despite the fact that the methods we propose can be applied to more complex problems (for example inhomogeneous media $[5]$ or the case of an impedance boundary condition on $\Gamma$ [21]). This is because we want to focus on analyzing the PML truncation and not on the rough surface itself. In addition the theoretical underpinnings are better understood and simpler for the sound soft case outlined above. So the choice of problem is for simplicity only since, as we shall see, the PML truncated variational scheme will converge whenever there is an appropriate variational formulation for the given rough surface problem which satisfies an inf-sup condition.

We first derive a basic estimate giving upper and lower bounds on the error in the approximation of the Dirichlet to Neumann map for a half space by the corresponding operator for a PML (see Theorem 3.1). 
This estimate can then be used to prove existence, uniqueness and convergence for a general rough surface satisfying (1.8). In particular, under appropriate conditions, we prove a first order convergence rate for the error in the global $V_{H}$ norm as the layer thickness increases (instead of the more standard exponential rate for bounded scatterers). By considering in detail scattering from a flat surface, we show that, in this norm, convergence cannot be faster than quadratic (up to logarithmic factors) due to the exponential damping of the PML solution along the surface. We also show that, for a half-space scatterer, exponential convergence is observed on compact subsets.

The analysis we shall give is just a first step. We shall use a PML only to truncate in the direction vertically away from the rough surface (for the use of an alternative method for truncation, the pole condition, and its relationship to (1.4) in the 2D case see [2]). A practical calculation also requires truncation laterally. This is true also for boundary integral equation approaches and is a well studied problem in that case (see for example [6]). We do not estimate the error from this truncation on the variational PML method. In addition we do not estimate the error in the resulting finite element scheme.

Our PML convergence proof suggests that, in the worst case, the method may converge slowly as the virtual thickness of the PML layer expands, and, in addition, it can be difficult to determine the optimal PML parameters in realistic simulations. We are thus motivated to combine the variational PML method with an iterative improvement scheme motivated by the work of Liu and Jin [18] using an integral operator with a smooth kernel to provide a correction to the variational PML scheme. We prove that this iterative approach converges. The cost of each iteration includes the cost of evaluating the integral operator which can be done rapidly using the Fast Fourier Transform since the integral operator in this case has a smooth kernel, and the solution of a finite element problem on a truncated portion of the strip $S_{H}$.

The outline of the paper is as follows. In the next section we recall the variational formulation of the rough surface problem in [4] and provide a variational formulation using the PML via a change of variables approach. Then in Section 3 we prove a general error estimate for the solution of the truncated PML problem using the variational formulation and a Fourier analysis of the PML layer. This result proves only first order convergence in the global $V_{H}$ norm. The sharpness of this convergence result is then investigated in Section 4 where we derive some estimates for the PML solution when the scatterer is flat. On the one hand, these estimates show that, even in this simple case, the PML solution does not converge exponentially rapidly to the exact solution in the global $V_{H}$ norm, since the PML solution does not have the right asymptotic behaviour at infinity. On the other hand, we show that on compact subsets of $S_{H}$ the PML solution does converge exponentially rapidly as the layer thickness increases. Our analysis of the flat scatterer, where we are able to obtain exact representations of the error in the PML approximation, is somewhat reminiscent of recent analysis of the time domain PML for simple geometries in [11]. In Section 5 we show how an iterative scheme to improve the PML solution can be constructed and prove its convergence using the estimates from Section 3. Finally, we present some very limited numerical examples testing our theory in Section 6 and finally draw some conclusions in Section 7 . We shall present the method and our analysis in $\mathbb{R}^{n}, n=2,3$, but the numerical results are in $\mathbb{R}^{2}$.

2. Variational Formulation and the PML. We start by recalling a variational formulation for the sound soft rough surface scattering problem used in [4]. In order to write down this variational formulation, we first define the appropriate Dirichlet-to-Neumann map $T: H^{1 / 2}\left(\Gamma_{H}\right) \rightarrow H^{-1 / 2}\left(\Gamma_{H}\right)$ for the domain $U_{H}$. Precisely, for a given function $\phi \in H^{1 / 2}\left(\Gamma_{H}\right)$, we have

$$
T=\mathcal{F}^{-1} M_{z} \mathcal{F}
$$

where $\mathcal{F}$ is the Fourier transform operator defined in (1.5) and $M_{z}$ is the operator in transform space of multiplication by $z(\xi)$ given by

$$
z(\xi)= \begin{cases}-\mathrm{i} \sqrt{k^{2}-\xi^{2}} & \text { for }|\xi| \leq k \\ \sqrt{\xi^{2}-k^{2}} & \text { for }|\xi|>k\end{cases}
$$

With this definition it can be shown that if $u$ satisfies (1.3), (1.4) and (1.6) in the sense described in the introduction then

$$
\frac{\partial u}{\partial \nu}=-T u \text { on } \Gamma_{H} .
$$


Using this boundary condition and standard variational arguments (see [4] for details) we can pose (1.3), (1.4) and (1.6) as the variational problem of finding the function $u \in V_{H}$ such that

$$
b(u, \phi)=-(g, \phi) \text { for all } \phi \in V_{H},
$$

where the sesquilinear form $b(.,$.$) is given by$

$$
b(u, \phi)=\int_{S_{H}}\left(\nabla u \cdot \nabla \bar{\phi}-k^{2} u \bar{\phi}\right) d x+\int_{\Gamma_{H}} \bar{\phi} T u d s
$$

and

$$
(g, \phi)=\int_{S_{H}} g \bar{\phi} d x .
$$

Recall that $H \geq f_{+}$is chosen so that the data $g \in L^{2}\left(S_{H}\right)$ has compact support in $S_{H}$. In [4] we show that the sound soft acoustic scattering problem given by (1.3), (1.4) and (1.6) is equivalent to (2.3) for all $k>0$ and with only the constraint (1.1) on $\Gamma$.

From the point of view of this paper, the key results from [4] are first that, under the boundary constraint (1.8), there is a constant $\gamma=\gamma\left(k, H, f_{-}\right)>0$ such that $b(.,$.$) satisfies the following inf-sup condition: for all$ $v \in V_{H}$

$$
\gamma\|v\|_{V_{H}} \leq \sup _{\phi \in V_{H}} \frac{|b(v, \phi)|}{\|\phi\|_{V_{H}}}
$$

We show in [4] that

$$
\gamma \geq 1 /\left(1+\sqrt{2} K(K+1)^{2}\right)
$$

where $K=k\left(H-f_{-}\right)$. Second, it follows from (2.5) (see [4] for details) that if $g \in V_{H}^{*}$ ( $V_{H}^{*}$ is the dual space of $V_{H}$ ), then the variational problem of finding $v \in V_{H}$ such that

$$
b(v, \phi)=g(\phi) \text { for all } \phi \in V_{H}
$$

has a unique solution that satisfies the a priori estimate

$$
\|v\|_{V_{H}} \leq \gamma^{-1}\|g\|_{V_{H}^{*}} .
$$

When we apply the PML, the standard Helmholtz equation (1.3) will be solved in $S_{H}$. Above $S_{H}$ we introduce another layer $S_{H}^{L}$ for some $L>0$ where the PML is located given by

$$
S_{H}^{L}=U_{H} \backslash \bar{U}_{H+L} .
$$

A summary of the various geometric objects in the paper is shown in Fig. 2.1.

The equations for the PML are derived by the change of variables technique [8,9]. In particular we introduce an integrable PML parameter $\sigma=\sigma\left(x_{n}\right)$ such that $\sigma\left(x_{n}\right)=1$ for $x_{n} \leq H$ and $\Re(\sigma)>0$ and $\Im(\sigma) \geq 0$. Then we can define the complex stretched coordinate $\hat{x}_{n}$ by

$$
\hat{x}_{n}=\int_{f_{-}}^{x_{n}} \sigma(t) d t+f_{-} .
$$

Clearly $\hat{x}_{n}=x_{n}$ for $x_{n} \leq H$. A typical choice of $\sigma$ is a power law given by

$$
\sigma(s)=\left\{\begin{array}{cl}
1 & \text { if } s \leq H \\
1+\sigma_{0}(s-H)^{m} & \text { if } s>H
\end{array}\right.
$$

where $\sigma_{0}$ is a complex constant and $m$ is a positive integer (we shall show results for $m=1$ ). An important parameter that governs the absorption of the PML is the virtual width of the layer denoted $\tilde{L}$ and given by

$$
\tilde{L}=\int_{H}^{H+L} \sigma(s) d s .
$$




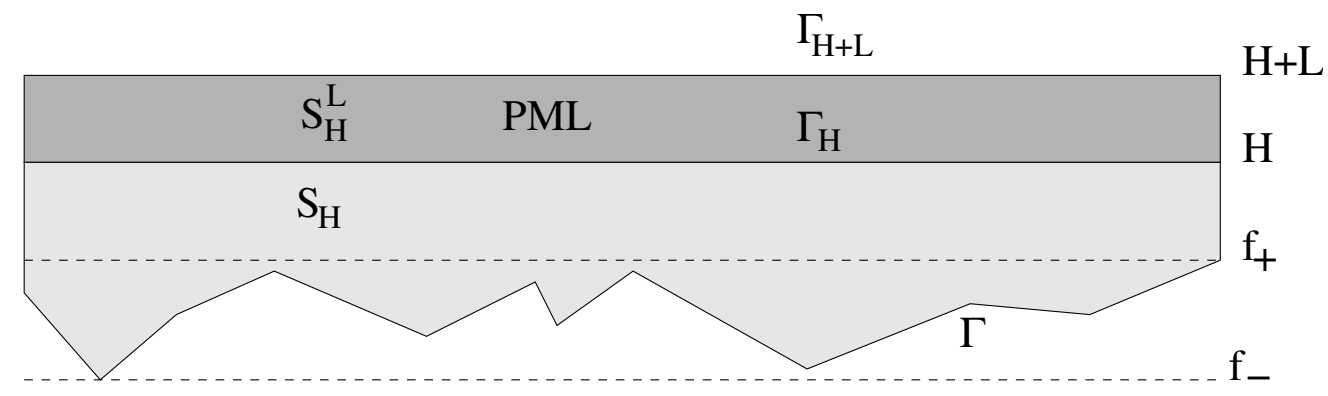

FIG. 2.1. A schematic showing some of the notation for the PML terminated acoustic rough surface problem. The lightly shaded region $S_{H}$ is the main computational domain where the Helmholtz equation is satisfied. The domain $S_{H}^{L}$ is occupied by the PML. A non-homogeneous Dirichlet boundary condition is applied on $\Gamma$ and a homogeneous Neumann condition is applied on $\Gamma_{H+L}$. The boundary $\Gamma_{H}$ is used in computing the Dirichlet to Neumann maps.

In general if the quantity $\Re(k \tilde{L})$ is large the PML will absorb evanescent modes well, whereas if $\Im(k \tilde{L})$ is large the PML will absorb waves propagating into the PML well.

Formally, the change of variables technique is to require that the solution in the PML, denoted $u_{p}$, satisfy the Helmholtz equation in stretched coordinates so

$$
\sum_{j=1}^{n-1} \frac{\partial^{2} u_{p}}{\partial x_{j}^{2}}+\frac{\partial^{2} u_{p}}{\partial \hat{x}_{n}^{2}}+k^{2} u_{p}=0 \text { in } S_{H}^{L} .
$$

Then changing variables back to standard real coordinates, using the fact that $d \hat{x}_{n} / d x_{n}=\sigma$ and the fact that $\sigma$ depends only on $x_{n}$, we obtain the PML equation

$$
\sum_{j=1}^{n-1} \frac{\partial}{\partial x_{j}}\left(\sigma \frac{\partial}{\partial x_{j}} u_{p}\right)+\frac{\partial}{\partial x_{n}}\left(\frac{1}{\sigma} \frac{\partial}{\partial x_{n}} u_{p}\right)+k^{2} \sigma u_{p}=0 \text { in } S_{H}^{L} .
$$

For convenience we then define the appropriate differential operator for the PML,

$$
\Delta_{p} v=\sum_{j=1}^{n-1} \frac{\partial}{\partial x_{j}}\left(\sigma \frac{\partial}{\partial x_{j}} v\right)+\frac{\partial}{\partial x_{n}}\left(\frac{1}{\sigma} \frac{\partial}{\partial x_{n}} v\right) .
$$

Of course $\Delta_{p}=\Delta$ outside the PML.

We can now state the truncated PML problem: we seek $u_{p} \in V_{H+L}$ such that

$$
\begin{aligned}
\Delta_{p} u_{p}+k^{2} \sigma u_{p} & =g \text { in } S_{H+L}, \\
\frac{\partial}{\partial x_{n}} u_{p} & =0 \text { on } \Gamma_{H+L},
\end{aligned}
$$

where (2.9) is understood in the sense of distributions and (2.10) by duality. The fact that $u_{p} \in V_{H+L}$ implies that $u_{p}=0$ on $\Gamma$. The choice of a Neumann condition on $\Gamma_{H+L}$ is essentially arbitrary and we shall compare this choice to the more standard choice of a Dirichlet boundary condition in Section 4.

For the purpose of analysis (for computational purposes the finite element grid covers all $S_{H+L}$ including the PML zone $S_{H}^{L}$ as well as $S_{H}$ ) we follow [7] and eliminate the PML by using the Dirichlet to Neumann map for the PML domain above $\Gamma_{H}$. For later use we consider a more general problem than is needed at this stage having non-homogeneous boundary data on $\Gamma_{H+L}$. In particular, given $s \in H^{-1 / 2}\left(\Gamma_{H+L}\right)$ and $q \in H^{1 / 2}\left(\Gamma_{H}\right)$ we wish to compute the Dirichlet data on $\Gamma_{H}$ for the problem of finding $v \in H^{1}\left(S_{H}^{L}\right)$ such that

$$
\begin{aligned}
v & =q \text { on } \Gamma_{H}, \\
\Delta_{p} v+k^{2} \sigma v & =0 \text { on } S_{H}^{L}, \\
\frac{1}{\sigma} \frac{\partial v}{\partial x_{n}} & =s \text { on } \Gamma_{H+L} . \\
5 &
\end{aligned}
$$


Since the solution in the PML zone $S_{H}^{L}$ is governed by a simple Helmholtz equation in stretched variables, we know that it will have standard plane wave solutions in the stretched coordinates. Using the plane wave solutions we see that if $\hat{v}\left(\xi, x_{n}\right)=\left(\mathcal{F} v\left(\cdot, x_{n}\right)\right)(\xi)$ then

$$
\hat{v}\left(\xi, x_{n}\right)=A \exp \left(z\left(\hat{x}_{n}-H\right)\right)+B \exp \left(-z\left(\hat{x}_{n}-H\right)\right) \text { for } H<x_{n}<H+L
$$

where $z=z(\xi)$ is given by (2.2). The Neumann boundary condition at $x_{n}=H+L$ implies that

$$
A \exp (z \tilde{L})-B \exp (-z \tilde{L})=\hat{s} / z .
$$

The requirement that $v=q$ when $x_{n}=H$ implies that $A+B=\hat{q}$ and thus, solving for $A$ and $B$, we obtain

$$
A=\frac{\hat{s} / z+\exp (-z \tilde{L}) \hat{g}}{\exp (z \tilde{L})+\exp (-z \tilde{L})} \text { and } B=\frac{-\hat{s} / z+\exp (z \tilde{L}) \hat{q}}{\exp (z \tilde{L})+\exp (-z \tilde{L})}
$$

Hence, for $H<x_{n}<H+L$,

$$
\begin{aligned}
\hat{v}= & \frac{\exp \left(z\left(\hat{x}_{n}-H\right)\right)-\exp \left(-z\left(\hat{x}_{n}-H\right)\right)}{z(\exp (z \tilde{L})+\exp (-z \tilde{L}))} \hat{s} \\
& +\frac{\exp \left(z\left(\hat{x}_{n}-H-\tilde{L}\right)\right)+\exp \left(-z\left(\hat{x}_{n}-H-\tilde{L}\right)\right)}{\exp (z \tilde{L})+\exp (-z \tilde{L})} \hat{q} .
\end{aligned}
$$

We want the Neumann data for the strip $S_{H}^{L}$ on $\Gamma_{H}$ so using the fact that $\sigma=1$ at $x_{n}=H$ we can evaluate

$$
-\left.\frac{\partial \hat{v}}{\partial x_{n}}\right|_{x_{n}=H}=\frac{-2}{\exp (z \tilde{L})+\exp (-z \tilde{L})} \hat{s}+z \frac{\exp (z \tilde{L})-\exp (-z \tilde{L})}{\exp (z \tilde{L})+\exp (-z \tilde{L})} \hat{q} .
$$

We therefore define the operators $T_{p}: H^{1 / 2}\left(\Gamma_{H}\right) \rightarrow H^{-1 / 2}\left(\Gamma_{H}\right)$ (this mapping property will be proved in Theorem 3.1) by

$$
\widehat{T_{p} q}=z \frac{\exp (z \tilde{L})-\exp (-z \tilde{L})}{\exp (z \tilde{L})+\exp (-z \tilde{L})} \hat{q}
$$

and $N_{p}: H^{-1 / 2}\left(\Gamma_{H+L}\right) \rightarrow H^{-1 / 2}\left(\Gamma_{H}\right)$ (this mapping property is a consequence of the estimates in Theorem 3.1) by

$$
\widehat{N_{p} s}=\frac{-2}{\exp (z \tilde{L})+\exp (-z \tilde{L})} \hat{s}
$$

and we have

$$
-\left.\frac{\partial v}{\partial x_{n}}\right|_{x_{n}=H}=T_{p} q+N_{p} s
$$

Returning now to the PML problem in which the Neumann data at $x_{n}=H+L$ is $s=0$, and using the inverse Fourier transform, the PML Dirichlet to Neumann map $T_{p}$ is given by

$$
\begin{aligned}
T_{p} u_{p}(\tilde{x}) & =-\frac{\partial u_{p}}{\partial x_{n}}(\tilde{x}, H) \\
& =\frac{1}{2 \pi} \int_{-\infty}^{\infty} z(\xi) \exp (-\mathrm{i} \xi \cdot \tilde{x})\left(\frac{\exp (z \tilde{L})-\exp (-z \tilde{L})}{\exp (z \tilde{L})+\exp (-z \tilde{L})}\right) \hat{u}_{p}(\xi, H) d \xi
\end{aligned}
$$

In terms of the Fourier transform operator $\mathcal{F}$ we see that

$$
T_{p}=\mathcal{F}^{-1} M_{z_{p}} \mathcal{F}
$$


where $M_{z_{p}}$ is the operator of multiplication by the function $z_{p}$ defined by

$$
z_{p}(\xi)=z(\xi)\left(\frac{\exp (z(\xi) \tilde{L})-\exp (-z(\xi) \tilde{L})}{\exp (z(\xi) \tilde{L})+\exp (-z(\xi) \tilde{L})}\right), \quad \xi \in \mathbb{R}^{n-1}
$$

Using $T_{p}$ we now find that the PML problem is equivalent to finding $u_{p} \in V_{H}$ such that

$$
b_{p}\left(u_{p}, \phi\right)=-(g, \phi) \text { for all } \phi \in V_{H},
$$

where

$$
b_{p}\left(u_{p}, \phi\right)=\int_{S_{H}}\left(\nabla u_{p} \cdot \nabla \bar{\phi}-k^{2} u_{p} \bar{\phi}\right) d x+\int_{\Gamma_{H}} \bar{\phi} T_{p} u_{p} d s
$$

The next section will be devoted to showing that (2.13) has a unique solution provided the real and imaginary parts of $\tilde{L}$ are large enough. We shall also prove an error estimate for the approximation of $u$ by $u_{p}$.

Before starting our analysis we need to define some more notation and norms. The space of bounded linear operators from a Hilbert space $X$ to a Hilbert space $Y$ is denoted by $\mathcal{L}(X, Y)$. We shall compute errors using various norms on the boundary $\Gamma_{H}$. It proves useful to define $H^{s}\left(\mathbb{R}^{n-1}\right)$ to be the Hilbert space of functions $\phi$ with finite $k$-dependent norm given by

$$
\|\phi\|_{H^{s}\left(\mathbb{R}^{n-1}\right)}^{2}=\int_{\mathbb{R}^{n-1}}|\hat{\phi}(\xi)|^{2}\left(k^{2}+\xi^{2}\right)^{s} d \xi
$$

This norm is equivalent to the standard norm on $H^{s}\left(\mathbb{R}^{n-1}\right)$ for fixed $k>0$. We need to estimate operator norms using this norm. In particular it is easy to verify that if $T$ is given by (2.1) then

$$
\|T\|_{\mathcal{L}\left(H^{1 / 2}\left(\mathbb{R}^{n-1}\right), H^{-1 / 2}\left(\mathbb{R}^{n-1}\right)\right)}=\sup _{\xi \in \mathbb{R}^{n-1}} \frac{|z(\xi)|}{\sqrt{k^{2}+\xi^{2}}}=1 .
$$

Norms of other operators defined by multiplication in the Fourier domain (for example $T_{p}$ and $T-T_{p}$ ) can be computed similarly.

3. Error estimates for the general truncated problem. The sesquilinear form $b(.,$.$) (see (2.4))$ defines an operator $B: V_{H} \rightarrow V_{H}^{*}$ by

$$
(B u, \phi)=b(u, \phi) \quad \text { for all } \phi \in V_{H},
$$

and the results of $[4]$ show that $B$ is invertible and

$$
\left\|B^{-1}\right\|_{\mathcal{L}\left(V_{H}^{*}, V_{H}\right)} \leq \frac{1}{\gamma}
$$

where $\gamma$ is the inf-sup constant in (2.5). In the same way the PML sesquilinear form $b_{p}(.,$.$) defines an$ operator $B_{p}: V_{H} \rightarrow V_{H}^{*}$ by

$$
\left(B_{p} u, \phi\right)=b_{p}(u, \phi) \text { for all } \phi \in V_{H} .
$$

Now defining $\delta B=B-B_{p}$ we see that

$$
\begin{aligned}
|(\delta B u, \phi)| & =\left|b(u, \phi)-b_{p}(u, \phi)\right| \\
& =\left|\int_{\Gamma_{H}} \bar{\phi}\left(T-T_{p}\right) u d s\right| \\
& \leq\|\phi\|_{H^{1 / 2}\left(\Gamma_{H}\right)}\left\|T-T_{p}\right\|_{\mathcal{L}\left(H^{1 / 2}\left(\Gamma_{H}\right), H^{-1 / 2}\left(\Gamma_{H}\right)\right)}\|u\|_{H^{1 / 2}\left(\Gamma_{H}\right)} \\
& \leq 2\left\|T-T_{p}\right\|_{\mathcal{L}\left(H^{1 / 2}\left(\Gamma_{H}\right), H^{-1 / 2}\left(\Gamma_{H}\right)\right)}\|u\|_{V_{H}}\|\phi\|_{V_{H}}
\end{aligned}
$$


where the last inequality follows from the trace theorem that

$$
\|\phi\|_{H^{1 / 2}\left(\Gamma_{H}\right)} \leq \sqrt{2}\|\phi\|_{V_{H}}
$$

for any $\phi \in V_{H}$ (proved in [4]). Standard operator perturbation theory [14] tells us that $B_{p}$ is invertible provided $\|\delta B\|_{\mathcal{L}\left(V_{H}, V_{H}^{*}\right)}<\gamma$. But, for $u \in V_{H}$,

$$
\|\delta B u\|_{V_{H}^{*}}=\sup _{\phi \in V_{H}} \frac{|(\delta B u, \phi)|}{\|\phi\|_{V_{H}}} \leq 2\left\|T-T_{p}\right\|_{\mathcal{L}_{\left(H^{1 / 2}\left(\Gamma_{H}\right), H^{-1 / 2}\left(\Gamma_{H}\right)\right)}\|u\|_{V_{H}} .}
$$

Thus

$$
\|\delta B\|_{\mathcal{L}\left(V_{H}, V_{H}^{*}\right)} \leq 2\left\|T-T_{p}\right\|_{\mathcal{L}\left(H^{1 / 2}\left(\Gamma_{H}\right), H^{-1 / 2}\left(\Gamma_{H}\right)\right)}
$$

and it suffices to prove that $T_{p} \rightarrow T$ in norm as the real and imaginary parts of $\tilde{L}$ tend to infinity appropriately. This is the content of the following fundamental theorem providing an error estimate for the approximation of the half-space Dirichlet to Neumann operator by a PML.

Theorem 3.1. Let $\alpha=\Re(k \tilde{L})$ and $\beta=\Im(k \tilde{L})$. Then

$$
C_{L}(\alpha, \beta) \leq\left\|T-T_{p}\right\|_{\mathcal{L}\left(H^{1 / 2}\left(\Gamma_{H}\right), H^{-1 / 2}\left(\Gamma_{H}\right)\right)} \leq C_{U}(\alpha, \beta),
$$

where

$$
C_{L}(\alpha, \beta)=\frac{1}{2 \sqrt{2} \mathrm{e}} \max \left(\frac{1}{\sqrt{\alpha^{2}+1 / 8}}, \frac{1}{\beta}\right),
$$

and

$$
C_{U}(\alpha, \beta)=\frac{1}{\mathrm{e}} \max \left\{\frac{1}{\alpha}+\frac{\beta}{\pi \alpha^{2}}, \frac{1}{\beta}+\frac{\alpha}{\pi \beta^{2}}\right\}= \begin{cases}\frac{1}{\mathrm{e} \alpha}+\frac{\beta}{\mathrm{e} \pi \alpha^{2}}, & \alpha \leq \beta \\ \frac{1}{\mathrm{e} \beta}+\frac{\alpha}{\mathrm{e} \pi \beta^{2}}, & \beta \leq \alpha\end{cases}
$$

REMARK 3.2. When $k|\tilde{L}|$ is large the lower bound $C_{L}(\alpha, \beta)$ is minimised approximately for fixed $k|\tilde{L}|$ by taking $\alpha=\beta$. With this choice the above theorem gives the estimate

$$
\frac{1}{2 \sqrt{2} \alpha \mathrm{e}} \leq\left\|T-T_{p}\right\|_{\mathcal{L}\left(H^{1 / 2}\left(\Gamma_{H}\right), H^{-1 / 2}\left(\Gamma_{H}\right)\right)} \leq \frac{\pi+1}{\mathrm{e} \pi \alpha} .
$$

Proof. Using the Fourier transform shows that

$$
\left\|T-T_{p}\right\|_{\mathcal{L}\left(H^{1 / 2}\left(\Gamma_{H}\right), H^{-1 / 2}\left(\Gamma_{H}\right)\right)}=\sup _{\xi \in \mathbb{R}} \frac{|z(\xi)|}{\sqrt{k^{2}+\xi^{2}}}|1-\tanh (z(\xi) \tilde{L})| .
$$

Using the fact that

$$
1-\tanh (z \tilde{L})=2 \frac{\exp (-2 z \tilde{L})}{1+\exp (-2 z \tilde{L})}
$$

we obtain

$$
\begin{aligned}
\left\|T-T_{p}\right\|_{\mathcal{L}\left(H^{1 / 2}\left(\Gamma_{H}\right), H^{-1 / 2}\left(\Gamma_{H}\right)\right)} & =\sup _{t \in \mathbb{R}} 2 \frac{\left|\sqrt{1-t^{2}}\right|}{\sqrt{1+t^{2}}} \frac{\left|\exp \left(2 \mathrm{i} \sqrt{1-t^{2}} k \tilde{L}\right)\right|}{\left|1+\exp \left(2 \mathrm{i} \sqrt{1-t^{2}} k \tilde{L}\right)\right|} \\
& =\max \left\{S_{1}, S_{2}\right\}
\end{aligned}
$$


where (defining $p=\sqrt{1-t^{2}}$ )

$$
\begin{aligned}
S_{1} & =\sup _{0 \leq t \leq 1} 2 \frac{\sqrt{1-t^{2}}}{\sqrt{1+t^{2}}} \frac{\exp \left(-2 \sqrt{1-t^{2}} \beta\right)}{\left|1+\exp \left(2 \mathrm{i} \sqrt{1-t^{2}} k \tilde{L}\right)\right|} \\
& =\sup _{0 \leq p \leq 1} \frac{2 p}{\sqrt{2-p^{2}}} \frac{\exp (-2 p \beta)}{|1+\exp (2 \mathrm{i} p k \tilde{L})|},
\end{aligned}
$$

and (now defining $p=\sqrt{t^{2}-1}$ )

$$
\begin{aligned}
S_{2} & =\sup _{t \geq 1} 2 \frac{\sqrt{t^{2}-1}}{\sqrt{1+t^{2}}} \frac{\exp \left(-2 \sqrt{t^{2}-1} \alpha\right)}{\left|1+\exp \left(2 \mathrm{i} \sqrt{t^{2}-1} k \tilde{L}\right)\right|} \\
& =\sup _{p \geq 0} \frac{2 p}{\sqrt{2+p^{2}}} \frac{\exp (-2 p \alpha)}{|1+\exp (-2 p k \tilde{L})|} .
\end{aligned}
$$

It remains to estimate $S_{1}$ and $S_{2}$.

We can easily verify that for any $\gamma>0$ the function $f(p)=p \exp (-2 p \gamma)$ attains its maximum value of $1 /(2 \gamma \mathrm{e})$ when $p=1 /(2 \gamma)$. Now consider $|1+\exp (-p(\mu+\mathrm{i} \nu))|$ for real parameters $\mu>0, \nu>0$ and $p \geq 0$. Clearly

$$
2 \geq|1+\exp (-p(\mu+\mathrm{i} \nu))| \geq \Re(1+\exp (-p(\mu+\mathrm{i} \nu)))=1+\exp (-p \mu) \cos (p \nu) .
$$

The extreme points of the function $g(p)=\exp (-p \mu) \cos (p \nu)$ are the points satisfying $\cos (\theta-p \nu)=0$, where $\theta:=\sin ^{-1}\left(\nu / \sqrt{\nu^{2}+\mu^{2}}\right)$. The global minimum of $1+\exp (-p \mu) \cos (p \nu)$ occurs at the first minimum where $p \nu-\theta=\pi / 2$. Thus

$$
2 \geq \mid 1+\exp (-p(\mu+i \nu) \mid \geq 1-F(\mu / \nu),
$$

where the function $F$ is defined by

$$
F(s):=\frac{\exp \left(-s\left(\pi / 2+\tan ^{-1}\left(s^{-1}\right)\right)\right)}{\sqrt{1+s^{2}}}, \quad s>0 .
$$

But, for $s>0$,

$$
\tan ^{-1}\left(s^{-1}\right)=\frac{\pi}{2}-\int_{1 / s}^{\infty} \frac{d t}{1+t^{2}} \geq \frac{\pi}{2}-\int_{1 / s}^{\infty} \frac{d t}{t^{2}} \geq \frac{\pi}{2}-s
$$

Thus

$$
(F(s))^{-1} \geq \exp \left(s\left(\pi / 2+\tan ^{-1}\left(s^{-1}\right)\right)\right) \geq 1+s\left(\pi / 2+\tan ^{-1}\left(s^{-1}\right)\right)+\frac{s^{2} \pi^{2}}{8} \geq 1+s \pi,
$$

so that $1-F(s) \geq s \pi /(1+s \pi)$ and

$$
2 \geq \mid 1+\exp \left(-p(\mu+i \nu) \mid \geq \frac{\mu \pi}{\nu+\mu \pi} .\right.
$$

Applying these results, to

$$
|1+\exp (2 \mathrm{i} p k \tilde{L})|=|1+\exp (-p(2 \beta-2 \mathrm{i} \alpha))|=|1+\exp (-p(2 \beta+2 \mathrm{i} \alpha))|
$$

and to $|1+\exp (-2 p k \tilde{L})|=|1+\exp (-p(2 \alpha+2 \mathrm{i} \beta))|$, shows that

$$
\begin{aligned}
\frac{1}{2 \beta \mathrm{e} \sqrt{2}} & \leq S_{1} \leq \frac{1}{\beta \mathrm{e}}\left(1+\frac{\alpha}{\pi \beta}\right), \\
\frac{1}{2 \alpha \mathrm{e} \sqrt{2+\frac{1}{4 \alpha^{2}}}} \leq S_{2} & \leq \frac{1}{\sqrt{2} \alpha \mathrm{e}}\left(1+\frac{\beta}{\pi \alpha}\right),
\end{aligned}
$$


from which the estimate follows. $\square$

Using the argument preceding this theorem, together with the results of the theorem, we have now proved that provided $C_{U}(\alpha, \beta)$ is sufficiently small, the PML truncated variational problem has a unique solution.

To provide an error estimate we use the inf-sup condition. Since

$$
b_{p}(u, \phi)=b(u, \phi)+\int_{\Gamma_{H}} \bar{\phi}\left(T_{p}-T\right) u d s
$$

we have, for any $u \in V_{H}$, using the trace estimate (3.1), that

$$
\sup _{v \in V_{H}} \frac{\left|b_{p}(u, v)\right|}{\|v\|_{V_{H}}} \geq \gamma\|u\|_{V_{H}}-2\left\|T-T_{p}\right\|_{\mathcal{L}\left(H^{1 / 2}\left(\Gamma_{H}\right), H^{-1 / 2}\left(\Gamma_{H}\right)\right)}\|u\|_{V_{H}} .
$$

From Theorem 3.1 we thus have

$$
\sup _{v \in V_{H}} \frac{\left|b_{p}(u, v)\right|}{\|v\|_{V_{H}}} \geq\left(\gamma-2 C_{U}(\alpha, \beta)\right)\|u\|_{V_{H}}
$$

and $\gamma-2 C_{U}(\alpha, \beta)>0$ if $\alpha$ and $\beta$ are chosen appropriately. Thus $b_{p}$ satisfies an inf-sup condition whenever $b(.,$.$) satisfies an inf-sup condition provided \tilde{L}$ is chosen such that $C_{U}(\alpha, \beta)$ is small enough.

The inf-sup condition allows us to prove an error estimate, since, by the inf-sup condition, the definitions of the sesquilinear forms, the variational equations satisfied by $u$ and $u_{p}$, and the trace estimate (3.1), we have

$$
\begin{aligned}
\left(\gamma-2 C_{U}(\alpha, \beta)\right)\left\|u-u_{p}\right\|_{V_{H}} & \leq \sup _{v \in V_{H}} \frac{\left|b_{p}\left(u-u_{p}, v\right)\right|}{\|v\|_{V_{H}}} \\
& =\sup _{v \in V_{H}} \frac{\left|\int_{\Gamma_{H}} \bar{v}\left(T_{p}-T\right) u d s\right|}{\|v\|_{V_{H}}} \\
& \leq \sqrt{2}\left\|\left(T-T_{p}\right) u\right\|_{H^{-1 / 2}\left(\Gamma_{H}\right)}
\end{aligned}
$$

We have thus proved the following theorem:

TheOREm 3.3. Suppose $D$ satisfies the boundary constraint (1.8) and that $2 C_{U}(\alpha, \beta)<\gamma$, where $\alpha=\Re(k \tilde{L}), \beta=\Im(k \tilde{L}), C_{U}(\alpha, \beta)$ is the upper bound appearing in Theorem 3.1, and $\gamma$ is the inf-sup constant for the sesquilinear form b(.,.) (bounded below in (2.6)). Then the PML truncated problem (2.13) has a unique solution $u_{p} \in V_{H}$. Furthermore

$$
\left\|u-u_{p}\right\|_{V_{H}} \leq \frac{\sqrt{2}}{\gamma-2 C_{U}(\alpha, \beta)}\left\|\left(T-T_{p}\right) u\right\|_{H^{-1 / 2}\left(\Gamma_{H}\right)},
$$

where $u \in V_{H}$ is the solution of (1.3)-(1.4).

Of course it is difficult to estimate $\left(T-T_{p}\right) u$ a priori, but it may be known, for example, that $u$ has negligible evanescent modes. This would guide the choice of $\tilde{L}$ and the ultimate estimate of the error. We shall use this theorem in the next section.

In general, using Theorem 3.1 and the above theorem, we have the following worst case estimate which requires no special properties for $u$.

COROLlary 3.4. If the conditions of Theorem 3.3 are satisfied then

$$
\left\|u-u_{p}\right\|_{V_{H}} \leq \frac{2 C_{U}(\alpha, \beta)}{\gamma-2 C_{U}(\alpha, \beta)}\|u\|_{V_{H}} .
$$

REMARK 3.5. If, for some constant $c>1$, it holds that $c^{-1} \leq \alpha / \beta \leq c$, then this corollary implies that

$$
\left\|u-u_{p}\right\|_{V_{H}}=O\left(\frac{1}{\alpha}\right)
$$


as $\alpha \rightarrow \infty$, which is much slower than the exponential rate of convergence proved in [16, 15, 7]. We shall, however, show in Section 4 that, even in the simplest case when the rough surface is flat, exponential convergence in the $\|\cdot\|_{V_{H}}$ norm is not achieved. In fact we shall see in Remark 4.5 that the estimate (3.4) is fairly sharp.

4. A special case: a flat surface. In this section we shall analyze the special case of scattering by a flat surface in $\mathbb{R}^{2}$ so that for this section $D=U_{0} \subset \mathbb{R}^{2}$ and $\Gamma=\Gamma_{0}$. Our goal is to obtain asymptotic estimates for the accuracy of the PML solution in two limits. The first is for a fixed PML as the lateral distance $\left|x_{1}\right| \rightarrow \infty$ and the second is for a fixed position as the imaginary part of $\tilde{L}$ increases. A corollary of the first estimate is a lower bound for the error in the PML solution in the $V_{H}$ norm showing that global exponential convergence does not take place. This result shows that our general theorem is correct in predicting a polynomial convergence rate. The second estimate shows that despite poor global convergence, exponential convergence is seen on compact subsets, at least for this special case.

We shall also consider both the Dirichlet and Neumann backed PML and show that the two behave similarly, so, for this problem, there is no disadvantage to using the more convenient Neumann backed PML.

We shall choose the special case of $g=-\delta_{y}$ in (1.3) where $\delta_{y}$ is the delta distribution at $y=\left(0, y_{2}\right)$ where $y_{2}>0$. Strictly this falls outside the variational theory of [4] but by using a scattered field formulation the problem can be brought within that theory. In any case it is easy to verify the following exact solution:

$$
u(x)=\frac{\mathrm{i}}{4}\left(H_{0}^{(1)}(k|x-y|)-H_{0}^{(1)}\left(k\left|x-y^{\prime}\right|\right)\right),
$$

where $y^{\prime}=\left(0,-y_{2}\right)$ and $H_{n}^{(1)}$ denotes the Hankel function of the first kind of order $n$. Defining $R^{\prime}=\left|x-y^{\prime}\right|$, $R=|x-y|$, and recalling that $H_{0}^{(1) \prime}=-H_{1}^{(1)}$, we see that

$$
u(x)=\frac{\mathrm{i}}{4} \int_{k R}^{k R^{\prime}} H_{1}^{(1)}(s) d s .
$$

It follows from this representation, and since [1]

$$
H_{1}^{(1)}(s) \sim \sqrt{\frac{2}{\pi s}} e^{\mathrm{i}(s-3 \pi / 4)},
$$

as $s \rightarrow+\infty$, that, as $\left|x_{1}\right| \rightarrow \infty$ with $x_{2}, y_{2}=O(1)$,

$$
u(x) \sim \frac{\mathrm{i}}{4} k\left(R^{\prime}-R\right) \sqrt{\frac{2}{\pi k R}} e^{\mathrm{i}(k R-3 \pi / 4)} \sim \frac{\exp \left(\mathrm{i} k\left|x_{1}\right|\right)}{2 \sqrt{\pi}\left(k\left|x_{1}\right|\right)^{3 / 2}}(1-\mathrm{i}) k^{2} x_{2} y_{2},
$$

where to obtain this last equation we use that $R=\left|x_{1}\right|+o(1)$ and that $R^{\prime}-R=4 x_{2} y_{2} /\left(R^{\prime}+R\right) \sim 2 x_{2} y_{2}\left|x_{1}\right|^{-1}$, as $\left|x_{1}\right| \rightarrow \infty$ with $x_{2}, y_{2}=O(1)$.

It will be convenient also to write (4.1) using the Fourier transform. From the Fourier transform of the Hankel function we find that

$$
u(x)=\left\{\begin{array}{lc}
\frac{1}{2 \pi} \int_{-\infty}^{\infty} \frac{\sin \left(\kappa x_{2}\right)}{\kappa} \exp \left(\mathrm{i}\left(\kappa y_{2}-\xi x_{1}\right)\right) d \xi, & 0 \leq x_{2}<y_{2}, \\
\frac{1}{2 \pi} \int_{-\infty}^{\infty} \frac{\sin \left(\kappa y_{2}\right)}{\kappa} \exp \left(\mathrm{i}\left(\kappa x_{2}-\xi x_{1}\right)\right) d \xi, & x_{2}>y_{2},
\end{array}\right.
$$

where $\kappa=\sqrt{k^{2}-\xi^{2}}, \Im(\kappa) \geq 0$.

We assume that the PML starts at a height $H \geq y_{2}$ so that the Helmholtz equation is satisfied for $0<x_{2}<y_{2}$. Then, using the plane wave solutions of the Helmholtz equation for $x_{2}<y_{2}$ and of the PML-Helmholtz equation for $x_{2}>y_{2}$, we obtain that if the PML is terminated by a Neumann boundary condition, the solution of (2.9)-(2.10), denoted in this section by $u_{p, n}$ to distinguish it from the solution with a Dirichlet upper boundary condition, is

$$
u_{p, n}(x)=\left\{\begin{array}{lc}
\frac{1}{2 \pi} \int_{-\infty}^{\infty} \frac{\sin \left(\kappa x_{2}\right)}{\kappa} \frac{\cos \left(\kappa\left(y_{2}-(\tilde{L}+H)\right)\right)}{\cos (\kappa(\tilde{L}+H))} \exp \left(-\mathrm{i} \xi x_{1}\right) d \xi, & 0 \leq x_{2}<y_{2}, \\
\frac{1}{2 \pi} \int_{-\infty}^{\infty} \frac{\sin \left(\kappa y_{2}\right)}{\kappa} \frac{\cos \left(\kappa\left(x_{2}-(\tilde{L}+H)\right)\right)}{\cos (\kappa(\tilde{L}+H))} \exp \left(-\mathrm{i} \xi x_{1}\right) d \xi, & y_{2}<x_{2} \leq H .
\end{array}\right.
$$


If we now replace (2.10) by the Dirichlet condition that $u_{p}=0$ at $x_{2}=L+H$ we obtain the following solution valid for $0 \leq x_{2}<y_{2}$ and denoted $u_{p, d}$ :

$$
u_{p, d}(x)= \begin{cases}\frac{1}{2 \pi} \int_{-\infty}^{\infty} \frac{\sin \left(\kappa x_{2}\right)}{\kappa} \frac{\sin \left(\kappa\left((\tilde{L}+H)-y_{2}\right)\right)}{\sin (\kappa(\tilde{L}+H))} \exp \left(-\mathrm{i} \xi x_{1}\right) d \xi, & 0 \leq x_{2}<y_{2}, \\ \frac{1}{2 \pi} \int_{-\infty}^{\infty} \frac{\sin \left(\kappa y_{2}\right)}{\kappa} \frac{\sin \left(\kappa\left((\tilde{L}+H)-x_{2}\right)\right)}{\sin (\kappa(\tilde{L}+H))} \exp \left(-\mathrm{i} \xi x_{1}\right) d \xi, & y_{2}<x_{2} \leq H\end{cases}
$$

We will find second representations for the exact solutions to the PML problems useful. If $\left|x_{1}\right|$ is large enough, precisely if $(H+\Re \tilde{L})\left|x_{1}\right|>\left(x_{2}+y_{2}\right) \Im \tilde{L}$, we can evaluate the integrals (4.4) and (4.5) exactly, as a residue series, by contour integration, e.g. by moving the path of integration to the path $\Im \xi=Y$, for some $Y<0$, and then letting $Y \rightarrow \infty$. The only singularities in the lower half-plane are simple poles. In the Dirichlet case (4.5) these are at $\xi=-k \beta_{n}, n \in \mathbb{N}$, where

$$
\alpha_{n}:=\frac{n \pi}{k(H+\tilde{L})}, \quad \beta_{n}:=\sqrt{1-\alpha_{n}^{2}},
$$

with $\Im \beta_{n}>0$, and we arrive at the representation

$$
u_{p, d}(x)=\frac{\mathrm{i}}{k(H+\tilde{L})} \sum_{n=1}^{\infty} \beta_{n}^{-1} \sin \left(k y_{2} \alpha_{n}\right) \sin \left(k x_{2} \alpha_{n}\right) \exp \left(\mathrm{i} k\left|x_{1}\right| \beta_{n}\right) .
$$

We note that this formula is well known as an exact expression for the Dirichlet Green's function for a duct in the case when $\tilde{L}=0$ (the PML layer is empty); see e.g. Linton [17]. Indeed, the representation (4.6) can most easily be derived by using this fact coupled with analytic continuation arguments. One argues that (4.6) and (4.5) coincide in the case that $\Im \tilde{L}=0$, since they can be viewed as different representations for the values in $0 \leq x_{2} \leq H$ of the standard Dirichlet Green's function for a duct of width $H+\tilde{L}$ when $\tilde{L}>0$ (the various representations for this Green's functions are derived in [17]). Then one argues that both representations are analytic as a function of $\tilde{H}:=H+\tilde{L}$ in the region of the complex plane given by $\Re \tilde{H}>0$ and $\Re \tilde{H}\left|x_{1}\right|>\left(x_{2}+y_{2}\right) \Im \tilde{H}>0$, and are continuous in the union of this region with that part of the positive real axis $0<\tilde{H}<\pi / k$. This implies, by the uniqueness of analytic continuation, that the representations coincide throughout this region.

Similarly to (4.6), for the Neumann case one has the representation

$$
u_{p, n}(x)=\frac{\mathrm{i}}{k(H+\tilde{L})} \sum_{n=0}^{\infty} \tilde{\beta}_{n}^{-1} \sin \left(k y_{2} \tilde{\alpha}_{n}\right) \sin \left(k x_{2} \tilde{\alpha}_{n}\right) \exp \left(\mathrm{i} k\left|x_{1}\right| \tilde{\beta}_{n}\right),
$$

where

$$
\tilde{\alpha}_{n}:=\frac{(n+1 / 2) \pi}{k(H+\tilde{L})}, \quad \tilde{\beta}_{n}:=\sqrt{1-\tilde{\alpha}_{n}^{2}} .
$$

As for the Dirichlet case, one can justify this formula by analytic continuation arguments, using the fact that (4.4) and (4.7) are well-defined and coincide when $\tilde{H}=H+\tilde{L}$ is real and small enough [17].

The proof of our first result, specifically the upper bound in the proof on $\left|P_{n}\right|$ and the asymptotic formulae for $\left|\Im \alpha_{n}\right|$ and $\Im \beta_{n}$, justify the statement that (4.6) and (4.7) are analytic as a function of $\tilde{H}$ in the region of the complex plane given by $\Re \tilde{H}>0$ and $\Re \tilde{H}\left|x_{1}\right|>\left(x_{2}+y_{2}\right) \Im \tilde{H}>0$.

LEMma 4.1. The series (4.6) and (4.7) converge for $(H+\Re \tilde{L})\left|x_{1}\right|>\left(x_{2}+y_{2}\right) \Im \tilde{L}>0$ (but not if $\left.(H+\Re \tilde{L})\left|x_{1}\right|<\left(x_{2}+y_{2}\right) \Im \tilde{L}\right)$. Moreover, for $k|H+\tilde{L}| \geq \pi$ and

$$
\frac{3 \sqrt{2} \pi(H+\Re \tilde{L})\left|x_{1}\right|}{16 k|H+\tilde{L}|^{2}} \geq \frac{|H+\tilde{L}|^{2}}{\pi \Im \tilde{L}}+x_{2}+y_{2},
$$

it holds that

$$
\begin{aligned}
&\left|u_{p, d}(x)\right| \leq \frac{e}{(e-1) \sqrt{2 \pi k \Im \tilde{L}}} \exp \left(\frac{\pi \Im \tilde{L}\left(x_{2}+y_{2}\right)}{|H+\tilde{L}|^{2}}-\frac{\pi^{2}(H+\Re \tilde{L}) \Im \tilde{L}\left|x_{1}\right|}{|H+\tilde{L}|^{3} \sqrt{\pi^{2}+k^{2}|H+\tilde{L}|^{2}}}\right) \\
& \leq \frac{1}{(e-1) \sqrt{2 \pi k \Im \tilde{L}}} \exp \left(-\frac{5 \sqrt{2} \pi^{2}(H+\Re \tilde{L}) \Im \tilde{L}\left|x_{1}\right|}{16 k|H+\tilde{L}|^{4}}\right), \\
& 12
\end{aligned}
$$


while, for $k|H+\tilde{L}| \geq \pi$ and

$$
\frac{3 \sqrt{5} \pi(H+\Re \tilde{L})\left|x_{1}\right|}{40 k|H+\tilde{L}|^{2}} \geq \frac{|H+\tilde{L}|^{2}}{\pi \Im \tilde{L}}+x_{2}+y_{2},
$$

it holds that

$$
\begin{aligned}
\left|u_{p, n}(x)\right| & \leq \frac{e^{2}}{(e-1) \sqrt{2 \pi k \Im \tilde{L}}} \exp \left(\frac{\pi \Im \tilde{L}\left(x_{2}+y_{2}\right)}{|H+\tilde{L}|^{2}}-\frac{\pi^{2}(H+\Re \tilde{L}) \Im \tilde{L}\left|x_{1}\right|}{|H+\tilde{L}|^{3} \sqrt{\pi^{2}+4 k^{2}|H+\tilde{L}|^{2}}}\right) \\
& \leq \frac{e}{(e-1) \sqrt{2 \pi k \Im \tilde{L}}} \exp \left(-\frac{\sqrt{5} \pi^{2}(H+\Re \tilde{L}) \Im \tilde{L}\left|x_{1}\right|}{8 k|H+\tilde{L}|^{4}}\right) .
\end{aligned}
$$

Proof. We give the detail of these estimates for the Dirichlet case. The Neumann case follows similarly. It is convenient to write $(k(H+\tilde{L}))^{-1}$ as

$$
\frac{1}{k(H+\tilde{L})}=p-\mathrm{i} q
$$

with $p>0$ and $q>0$. Let $P_{n}=\sin \left(k y_{2} \alpha_{n}\right) \sin \left(k x_{2} \alpha_{n}\right) \exp \left(\mathrm{i} k\left|x_{1}\right| \beta_{n}\right)$, so that $P_{n} / \beta_{n}$ is the $n$th term in the series (4.6). For $Z=X+\mathrm{i} Y$, with $X, Y \in \mathbb{R}$, it holds that

$$
\frac{1}{2}\left(1-e^{-2|Y|}\right) e^{|Y|} \leq|\sin Z| \leq e^{|Y|} .
$$

Thus

$$
\frac{1}{4}\left(1-\exp \left(-2 k\left(x_{2}+y_{2}\right)\left|\Im \alpha_{n}\right|\right) \exp Q_{n} \leq\left|P_{n}\right| \leq \exp Q_{n}\right.
$$

where

$$
Q_{n}=k\left(x_{2}+y_{2}\right)\left|\Im \alpha_{n}\right|-k\left|x_{1}\right| \Im \beta_{n} .
$$

Now

$$
\left|\alpha_{n}\right|=n \pi \sqrt{p^{2}+q^{2}}, \quad\left|\Im \alpha_{n}\right|=n \pi q,
$$

and, as $n \rightarrow \infty$,

$$
\left|\beta_{n}\right|=\left|\alpha_{n}\right|+o(1), \quad \Im \beta_{n}=n \pi p+o(1) .
$$

From these estimates and (4.14) it is clear that (4.6) converges if $\left|x_{1}\right| p>\left(x_{2}+y_{2}\right) q$ but not if $\left|x_{1}\right| p<\left(x_{2}+y_{2}\right) q$.

To derive accurate estimates of the exponential decay of $u_{p, d}(x)$ for $\left|x_{1}\right|$ large, we need sharp bounds on $\left|P_{n}\right|$. In particular we need bounds that are sharp for small $n$, since the earlier terms in the series decay more slowly at infinity. Further, if we are interested particularly in convergence as the PML parameter $|\tilde{L}| \rightarrow \infty$, we need estimates which are sharp for the case when $p$ and $q$ are small. We calculate that

$$
\Im \beta_{n}=\frac{Y}{\sqrt{2} \sqrt{R+X}}=\frac{\sqrt{2} p q n^{2} \pi^{2}}{\sqrt{R+X}},
$$

where $X$ and $Y$ are the real and imaginary parts of $\beta_{n}^{2}=1-\alpha_{n}^{2}$, and where $R=\sqrt{X^{2}+Y^{2}}=\left|\beta_{n}\right|^{2}$. Explicitly,

$$
X=1+\left(q^{2}-p^{2}\right) n^{2} \pi^{2}, \quad Y=2 p q n^{2} \pi^{2},
$$


and

$$
R^{2}=\left(1-n^{2} \pi^{2}\left(q^{2}+p^{2}\right)\right)^{2}+4 n^{2} \pi^{2} q^{2}=\left(1-\left|\alpha_{n}\right|^{2}\right)^{2}+4 n^{2} \pi^{2} q^{2} \leq\left(1-\left|\alpha_{n}\right|^{2}\right)^{2}+4\left|\alpha_{n}\right|^{2}=\left(1+\left|\alpha_{n}\right|^{2}\right)^{2}
$$

Thus, for $p, q$, and $n$ all small,

$$
\Im \beta_{n} \approx p q n^{2} \pi^{2}
$$

while, for all values of $n, p$ and $q$,

$$
\Im \beta_{n} \geq \frac{p q n^{2} \pi^{2}}{\sqrt{R}} \geq \delta_{n}:=\frac{p q n^{2} \pi^{2}}{\sqrt{1+\left|\alpha_{n}\right|^{2}}}=\frac{p q n^{2} \pi^{2}}{\sqrt{1+n^{2} \pi^{2}\left(p^{2}+q^{2}\right)}} .
$$

Now

$$
\begin{aligned}
\frac{\delta_{n+1}-\delta_{n}}{p q \pi^{2}} & =\frac{(n+1)^{2} \sqrt{1+n^{2} \pi^{2}\left(p^{2}+q^{2}\right)}-n^{2} \sqrt{1+(n+1)^{2} \pi^{2}\left(p^{2}+q^{2}\right)}}{\sqrt{1+(n+1)^{2} \pi^{2}\left(p^{2}+q^{2}\right)} \sqrt{1+n^{2} \pi^{2}\left(p^{2}+q^{2}\right)}} \\
& \geq \frac{(n+1)^{4}\left(1+n^{2} \pi^{2}\left(p^{2}+q^{2}\right)\right)-n^{4}\left(1+(n+1)^{2} \pi^{2}\left(p^{2}+q^{2}\right)\right)}{2(n+1)^{2}\left(1+(n+1)^{2} \pi^{2}\left(p^{2}+q^{2}\right)\right) \sqrt{1+n^{2} \pi^{2}\left(p^{2}+q^{2}\right)}} \\
& =\frac{(2 n+1) n^{2}\left(2+(n+1)^{2} \pi^{2}\left(p^{2}+q^{2}\right)\right)}{2(n+1)^{2}\left(1+(n+1)^{2} \pi^{2}\left(p^{2}+q^{2}\right)\right) \sqrt{1+n^{2} \pi^{2}\left(p^{2}+q^{2}\right)}} \\
& \geq \frac{3 n}{8 \sqrt{1+n^{2} \pi^{2}\left(p^{2}+q^{2}\right)}} \geq \frac{3}{8 \sqrt{1+\pi^{2}\left(p^{2}+q^{2}\right)}},
\end{aligned}
$$

It follows that

$$
\delta_{n} \geq \delta_{1}+(n-1) \frac{3 p q \pi^{2}}{8 \sqrt{1+\pi^{2}\left(p^{2}+q^{2}\right)}}
$$

and so

$$
Q_{n} \leq k\left(x_{2}+y_{2}\right) \pi q-\frac{k\left|x_{1}\right| p q \pi^{2}}{\sqrt{1+\pi^{2}\left(p^{2}+q^{2}\right)}}+(n-1) \Psi=-\frac{5 k\left|x_{1}\right| p q \pi^{2}}{8 \sqrt{1+\pi^{2}\left(p^{2}+q^{2}\right)}}+n \Psi
$$

where

$$
\Psi:=k\left(x_{2}+y_{2}\right) \pi q-\frac{3 k\left|x_{1}\right| p q \pi^{2}}{8 \sqrt{1+\pi^{2}\left(p^{2}+q^{2}\right)}}
$$

Noting also that, from (4.15),

$$
\left|\beta_{n}\right|=\sqrt{R} \geq \sqrt{2 \pi q n} \geq \sqrt{2 \pi q}
$$

we see that the bound (4.9) holds for $\Psi \leq-1$. Moreover, it is easy to see that $\Psi \leq-1$ if $k|H+\tilde{L}| \geq \pi$ and (4.8) holds. Further, (4.10) holds if $\Psi \leq-1$ and $k|H+\tilde{L}| \geq \pi$. $\square$

The above result shows that both the Neumann-PML or Dirichlet-PML solutions decay exponentially as $\left|x_{1}\right| \rightarrow \infty$ and so do not capture the correct asymptotic behavior given by (4.2). This is hardly surprising and is not necessarily a drawback to the PML since the acoustic field may only be of interest in a bounded region near the source. But, to assess the sharpness of the upper bounds, on the difference between the true solution and the PML solution in the $V_{H}$ norm, that we obtained in Corollary 3.4 for the general rough surface case, it is of interest to estimate the impact that this inaccurate capture of the asymptotics at infinity has by obtaining lower bounds on $\left\|u-u_{p, d}\right\|_{V_{H}}$ and $\left\|u-u_{p, n}\right\|_{V_{H}}$.

Defining, for $v \in L^{2}\left(S_{H}\right)$ and $A \geq 0$,

$$
\|v\|_{2, A}=\left\{\int_{S_{H, A}}|v|^{2} d x\right\}^{1 / 2}
$$


where $S_{H, A}:=\left\{x \in S_{H}:\left|x_{1}\right| \geq A\right\}$, we have that

$$
\left\|u-u_{p, d}\right\|_{V_{H}} \geq k\left\|u-u_{p, d}\right\|_{2,0} \geq k\left\|u-u_{p, d}\right\|_{2, A} \geq k\|u\|_{2, A}-k\left\|u_{p, d}\right\|_{2, A},
$$

for every $A \geq 0$. Examining the derivation of (4.2), we see that this approximation is accurate provided $k\left|x_{1}\right| \gg 1$ and $k\left(R^{\prime}-R\right) \ll 1$; this certainly holds for $x \in S_{H, A}$ if $k A \gg 1$ and $k A \gg(k H)^{2}$. Thus, if $k A$ and $k A /(k H)^{2}$ are large enough then, from (4.2), for $x \in S_{H, A}$,

$$
|u(x)| \geq \frac{k^{1 / 2} x_{2} y_{2}}{\sqrt{3 \pi}\left|x_{1}\right|^{3 / 2}},
$$

so that

$$
\|u\|_{2, A} \geq \frac{(2 k)^{1 / 2} y_{2}}{\sqrt{3 \pi}}\left\{\int_{0}^{H} x_{2}^{2} d x_{2} \int_{A}^{\infty} x_{1}^{-3} d x_{1}\right\}^{1 / 2}=\frac{k^{1 / 2} y_{2} H^{3 / 2}}{3 \sqrt{\pi} A} .
$$

On the other hand, if $k|H+\tilde{L}| \geq \pi$ and

$$
\frac{3 \sqrt{2} \pi(H+\Re \tilde{L}) A}{16 k|H+\tilde{L}|^{2}} \geq \frac{|H+\tilde{L}|^{2}}{\pi \Im \tilde{L}}+2 H,
$$

then, applying Lemma 4.1, we see that (4.10) holds for $x \in S_{H, A}$, and so, where

$$
\theta:=\frac{5 \sqrt{2} \pi^{2}(H+\Re \tilde{L}) \Im \tilde{L}}{16 k|H+\tilde{L}|^{4}},
$$

we have that

$$
\left\|u_{p, d}\right\|_{2, A} \leq \frac{H^{1 / 2}}{(e-1) \sqrt{\pi k \Im \tilde{L}}}\left\{\int_{A}^{\infty} e^{-2 \theta x_{1}} d x_{1}\right\}^{1 / 2}=\frac{H^{1 / 2} e^{-\theta A}}{\sqrt{2 \pi}(e-1)(k \theta \Im \tilde{L})^{1 / 2}} .
$$

Combining these estimates with (4.16), we see that if $k A$ and $k A /(k H)^{2}$ are large enough, $k|H+\tilde{L}| \geq \pi$, (4.18) holds, and

$$
\frac{H^{1 / 2} e^{-\theta A}}{\sqrt{2 \pi}(e-1)(k \theta \Im \tilde{L})^{1 / 2}} \leq \frac{k^{1 / 2} y_{2} H^{3 / 2}}{6 \sqrt{\pi} A}
$$

then

$$
\left\|u-u_{p, d}\right\|_{V_{H}} \geq \frac{k^{3 / 2} y_{2} H^{3 / 2}}{6 \sqrt{\pi} A} .
$$

To make this estimate more explicit it remains to choose a value of $A$ to substitute in the above equation which satisfies the various constraints under which (4.21) is valid. Now our interest is in convergence as the dimensionless PML parameter, $k|\tilde{L}| \rightarrow \infty$. Clearly, in this limit, $k|H+\tilde{L}| \geq \pi$. Further, we note that (4.20) can be written as

$$
\theta A e^{-\theta A} \leq B^{-1}
$$

where

$$
B:=\frac{c^{*} k^{1 / 2}|H+\tilde{L}|^{6}}{y_{2} H(H+\Re \tilde{L})^{3 / 2}(\Im \tilde{L})^{2}} \geq c^{*}(k|H+\tilde{L}|)^{1 / 2}, \quad c^{*}:=\frac{5^{3 / 2} 2^{1 / 4} \pi^{3}(e-1)}{192} \approx 3.7,
$$

while (4.18) can be written as

$$
\theta A \geq \frac{5}{3}+\frac{10 \pi H \Im \tilde{L}}{3|H+\tilde{L}|^{2}} .
$$


We note that $B \rightarrow \infty$ as $k|\tilde{L}| \rightarrow \infty$. Moreover, defining

$$
A:=2 \theta^{-1} \log B
$$

we see that, for $k|\tilde{L}| \gg 1$, both (4.22) and (4.24) are satisfied and, moreover $k A \gg 1$ and $k A \gg(k H)^{2}$. Thus we have shown that (4.21) holds with $A$ given by (4.25) if $k|\tilde{L}|$ is sufficiently large, i.e. we have shown the following bound on $u-u_{p, d}$. The bound in this theorem on $u-u_{p, n}$ follows completely analogously, with minor changes to numerical values.

TheOrem 4.2. Provided $k|\tilde{L}|$ is sufficiently large, it holds that

$$
\left\|u-u_{p, d}\right\|_{V_{H}} \geq \frac{\hat{c} k^{1 / 2} y_{2} H^{3 / 2}(H+\Re \tilde{L}) \Im \tilde{L}}{|H+\tilde{L}|^{4} \log B},
$$

where $B$ is given by (4.23) and $\hat{c}:=\frac{5 \sqrt{2} \pi^{3 / 2}}{192} \approx 0.2$. The same lower bound holds for $\left\|u-u_{p, n}\right\|_{V_{H}}$, except that the constant $\hat{c}$ has the value $\frac{5 \sqrt{5} \pi^{3 / 2}}{96}$ and, in the definition of $B$, the constant $c^{*}$ has the value $5^{3 / 4} \pi^{3}(e-$ 1)/(96e).

It is interesting to compare the lower bound on $\left\|u-u_{p, n}\right\|_{V_{H}}$ in the above result with the upper bounds we have obtained already, for the general rough surface case, in Section 3. In particular, we see in the next lemma that this is a case where the bound in Theorem 3.3 is computable.

Lemma 4.3. Suppose that, for some constant $c>1$, it holds that $c^{-1} \leq \Re \tilde{L} / \Im \tilde{L} \leq c$. Then, where $T$ and $T_{p}$ are as defined in Section 3, it holds, for every $C>1$, that

$$
\left\|\left(T-T_{p}\right) u\right\|_{H^{-1 / 2}\left(\Gamma_{H}\right)} \leq C \frac{\sqrt{6} y_{2}(1+c \pi)}{8 \pi^{3 / 2} k} \sqrt{\frac{1}{(\Im \tilde{L})^{4}}+\frac{1}{(\Re \tilde{L})^{4}}}
$$

if $k|\tilde{L}|$ is sufficiently large.

Proof. Let $\psi=\left.u\right|_{\Gamma_{H}} \in H^{1 / 2}\left(\Gamma_{H}\right)=H^{1 / 2}(\mathbb{R})$ and $\phi=\left(T-T_{p}\right) \psi \in H^{-1 / 2}\left(\Gamma_{H}\right)=H^{-1 / 2}(\mathbb{R})$. Then, from (2.1) and (2.11), it follows that

$$
\hat{\phi}=\mathcal{F}\left(T-T_{p}\right) \psi=\left(z-z_{p}\right) \hat{\psi}
$$

and

$$
E:=\left\|\left(T-T_{p}\right) u\right\|_{H^{-1 / 2}\left(\Gamma_{H}\right)}=\left\{\int_{-\infty}^{\infty} \frac{\left|z(\xi)-z_{p}(\xi)\right|^{2}}{\sqrt{k^{2}+\xi^{2}}}|\hat{\psi}(\xi)|^{2} d \xi\right\}^{1 / 2} .
$$

Further, we can read off from (4.3) that

$$
\hat{\psi}=\frac{1}{\sqrt{2 \pi}} \frac{\sin \left(\kappa y_{2}\right)}{\kappa} \exp (\mathrm{i} \kappa H)
$$

and the definitions of $z$ and $z_{p}$ imply that $\left|z-z_{p}\right|=2|z||\exp (-2 z \tilde{L})| /|1+\exp (-2 z \tilde{L})|$. Noting also that $|z|=|\kappa|$, we see that

$$
E^{2}=\frac{4}{\pi} \int_{0}^{\infty} \frac{|\exp (-4 z \tilde{L})|}{|1+\exp (-2 z \tilde{L})|^{2}} \frac{\left|\sin ^{2}\left(\kappa y_{2}\right)\right|}{\sqrt{k^{2}+\xi^{2}}}|\exp (2 \mathrm{i} \kappa H)| d \xi=\frac{4}{\pi}\left(I_{1}+I_{2}\right)
$$

where, defining $\alpha=k \Re \tilde{L}, \beta=k \Im \tilde{L}$,

$$
\begin{aligned}
& I_{1}=\int_{0}^{1} \frac{\exp \left(-4 \sqrt{1-t^{2}} \beta\right)}{\left|1+\exp \left(2 \mathrm{i} \sqrt{1-t^{2}} \tilde{L}\right)\right|^{2}} \frac{\sin ^{2}\left(\sqrt{1-t^{2}} k y_{2}\right)}{\sqrt{1+t^{2}}} d t \\
& I_{2}=\int_{1}^{\infty} \frac{\exp \left(-4 \sqrt{t^{2}-1} \alpha\right)}{\left|1+\exp \left(-2 \sqrt{t^{2}-1} \tilde{L}\right)\right|^{2}} \frac{\sinh ^{2}\left(\sqrt{t^{2}-1} k y_{2}\right) \exp \left(-2 \sqrt{t^{2}-1} k H\right)}{\sqrt{1+t^{2}}} d t .
\end{aligned}
$$




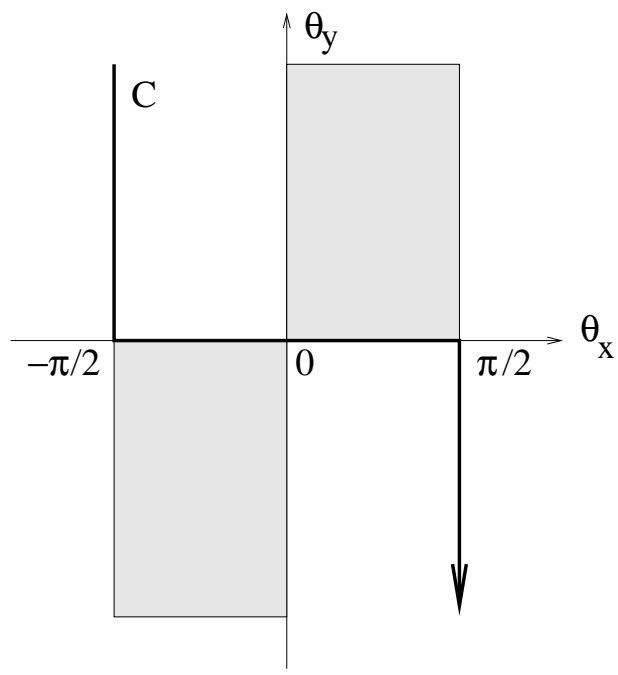

FIG. 4.1. A schematic showing the contour $C$ in the complex $\theta$ plane $\left(\theta=\theta_{x}+\mathrm{i} \theta_{y}\right)$ together with the unbounded regions (shaded) in which the poles of the integrand in $-\pi / 2 \leq \theta_{x} \leq \pi / 2$ may lie.

Now, applying (3.2), as at the end of the proof of Theorem 3.1, we see that $\left|1+\exp \left(2 \mathrm{i} \sqrt{1-t^{2}} \tilde{L}\right)\right|^{-1} \leq$ $(1+c \pi) / \pi$ and $\left|1+\exp \left(-2 \sqrt{t^{2}-1} \tilde{L}\right)\right|^{-1} \leq(1+c \pi) / \pi$. Thus, and making the change of variable $p=\sqrt{1-t^{2}}$ in $I_{1}$ and $p=\sqrt{t^{2}-1}$ in $I_{2}$, and noting that $\sinh u \leq u e^{u}$, for $u>0$, we see that $I_{1} \leq(1+c \pi)^{2} \tilde{I}_{1} / \pi^{2}$ and $I_{2} \leq(1+c \pi)^{2} \tilde{I}_{2} / \pi^{2}$ where

$\tilde{I}_{1}=k^{2} y_{2}^{2} \int_{0}^{1} \frac{p^{3} \exp (-4 p \beta)}{\sqrt{1-p^{2}}} d p, \quad \tilde{I}_{2}=k^{2} y_{2}^{2} \int_{0}^{\infty} p^{3} \exp \left(-p\left(4 \alpha+2 k\left(H-y_{2}\right)\right)\right) d p \leq k^{2} y_{2}^{2} \int_{0}^{\infty} p^{3} \exp (-4 p \alpha) d p$.

Thus $\tilde{I}_{2} \leq 6 k^{2} y^{2} /(4 \alpha)^{4}$, and $\tilde{I}_{1} \sim 6 k^{2} y^{2} /(4 \beta)^{4}$ as $\beta \rightarrow \infty$, from which the result follows.

Combining this lemma with Theorem 3.3 we obtain the following result.

COROLlary 4.4. Suppose that, for some constant $c>1$, it holds that $c^{-1} \leq \Re \tilde{L} / \Im \tilde{L} \leq c$. Then, for every $C>1$, it holds that

$$
\left\|u-u_{p, n}\right\|_{V_{H}} \leq C \frac{\sqrt{3} y_{2}(1+c \pi)}{4 \pi^{3 / 2} k \gamma} \sqrt{\frac{1}{(\Im \tilde{L})^{4}}+\frac{1}{(\Re \tilde{L})^{4}}},
$$

if $k|\tilde{L}|$ is sufficiently large, where $\gamma$ is the inf-sup constant of the sesquilinear form $b(\cdot, \cdot)$ (which satisfies $\gamma^{-1} \leq 1+\sqrt{2} k H(1+k H)^{2}$ by (2.6)).

REMARK 4.5. The estimates in Theorem 4.2 show that $\left\|u-u_{p, d}\right\|_{V_{H}}$ and $\left\|u-u_{p, n}\right\|_{V_{H}}$ decrease no faster than $O\left(|\tilde{L}|^{-3}(\log |\tilde{L}|)^{-1}\right)$ as $|\tilde{L}| \rightarrow \infty$, indeed no faster than $O\left(|\tilde{L}|^{-2}(\log |\tilde{L}|)^{-1}\right)$ as $|\tilde{L}| \rightarrow \infty$ if the ratio $\Im \tilde{L} / \Re \tilde{L}$ is bounded above and below. Complementing this, the estimate in the above corollary shows that $\left\|u-u_{p, n}\right\|_{V_{H}}=O\left(|\tilde{L}|^{-2}\right)$ as $|\tilde{L}| \rightarrow \infty$ if the ratio $\Im \tilde{L} / \Re \tilde{L}$ is bounded above and below. Thus, at least in the case that $\Re \tilde{L}$ and $\Im \tilde{L}$ increase approximately in proportion, we have identified, to within logarithmic factors, the rate of decrease of $\left\|u-u_{p, n}\right\|_{V_{H}}$ as $|\tilde{L}|$ increases, for the special case of the flat surface in $2 D$. This example shows us first that, because the PML solution fails to capture the correct asymptotics of the true solution at infinity, indeed decays exponentially fast, we cannot expect exponential convergence as $|\tilde{L}| \rightarrow \infty$, at least in the $\|\cdot\|_{V_{H}}$ norm. Secondly, it exhibits an example in which the bound in Theorem 3.3 for the general rough surface case is sharp, to within logarithmic factors, in its dependence on $|\tilde{L}|$. Finally, we note that we have seen already in Corollary 3.4 a very explicit upper bound on $\left\|u-u_{p, n}\right\|_{V_{H}}$, which applies in the general rough surface case, and which implies that $\left\|u-u_{p, n}\right\|_{V_{H}}=O\left(|\tilde{L}|^{-1}\right)$ as $|\tilde{L}| \rightarrow \infty$, provided the ratio $\Im \tilde{L} / \Re \tilde{L}$ is bounded above and below. We see that, in this $2 D$ flat surface case, this explicit bound underestimates the convergence as $|\tilde{L}| \rightarrow \infty$ by at most a power of $|\tilde{L}|$ (plus logarithmic factors). 
Our next result is more optimistic than the previous ones. For any fixed point $x=\left(x_{1}, x_{2}\right)$ we prove exponential convergence of the PML solution to the exact solution.

TheOREM 4.6. If $x=\left(x_{1}, x_{2}\right)$ satisfies $0 \leq x_{2} \leq H$ and $\left|x_{1}\right|<2 \Im(\tilde{L})$, then

$$
\left|u(x)-u_{p, d}(x)\right| \leq \frac{\exp (-2 k \Im(\tilde{L}))}{1-\exp (-2 k \Im(\tilde{L}))} \frac{1}{\sqrt{\pi k \Im(\tilde{L})}}\left(\frac{1}{2}+\frac{\exp \left(x_{1}^{2} /\left(4 \Im(\tilde{L})-2 x_{1}\right)\right)}{\sqrt{1-x_{1} /(2 \Im(\tilde{L}))}}\right) .
$$

The error $\left|u(x)-u_{p, n}(x)\right|$ satisfies the same bound.

REMARK 4.7. This theorem implies that, for every $x=\left(x_{1}, x_{2}\right)$ with $0 \leq x_{2} \leq H$ and for every $C>3 / 2$, it holds that

$$
\left|u(x)-u_{p, d}(x)\right| \leq C \sqrt{\frac{1}{\pi k \Im(\tilde{L})}} \exp (-2 k \Im(\tilde{L})),
$$

for all sufficiently large $\Im(\tilde{L})$. Further, the error $\left|u(x)-u_{p, n}(x)\right|$ satisfies the same bound.

A more careful analysis shows that this bound holds for every $C>1$ if $\Im(\tilde{L})$ is sufficiently large. In fact, from (4.32) below we see, using standard arguments for asymptotic estimates for integrals, that, as $\Im(\tilde{L}) \rightarrow \infty$ with $x$ fixed,

$$
\begin{aligned}
u(x)-u_{p, d}(x) & \sim \sin \left(k x_{2}\right) \sin \left(k y_{2}\right) \exp (2 \mathrm{i} k(\tilde{L}+H)) \frac{-\sqrt{2}}{\pi} \int_{-\infty}^{\infty} \exp \left(2 \mathrm{i} k(\tilde{L}+H) u^{2}\right) d u \\
& =-\sin \left(k x_{2}\right) \sin \left(k y_{2}\right) \frac{\exp (2 \mathrm{i} k(\tilde{L}+H))}{\sqrt{-\pi \mathrm{i} k(\tilde{L}+H)}},
\end{aligned}
$$

where to obtain this last equation we use the analytic continuation of (4.33) to the right hand complex plane, and the square root above is to be taken with positive real part. Thus

$$
\left|u(x)-u_{p, d}(x)\right| \sim\left|\sin \left(k x_{2}\right) \sin \left(k y_{2}\right)\right| \frac{\exp (-2 k \Im \tilde{L})}{\sqrt{\pi k|\tilde{L}+H|}} \leq \frac{\exp (-2 k \Im \tilde{L})}{\sqrt{\pi k \Im \tilde{L}}},
$$

as $|\Im \tilde{L}| \rightarrow \infty$. The identical asymptotic expression for $\left|u(x)-u_{p, n}(x)\right|$ follows from (4.34).

Proof. We shall give details for the Dirichlet-PML condition and a brief summary of the derivation in the case of the Neumann-PML. Subtracting (4.5) from (4.3) we obtain, in the case $0 \leq x_{2}<y_{2}$,

$$
u(x)-u_{p, d}(x)=\frac{1}{2 \pi} \int_{-\infty}^{\infty} \exp \left(\mathrm{i}\left(\kappa y_{2}-\xi x_{1}\right)\right) \frac{\sin \left(\kappa x_{2}\right)}{\kappa} e_{D} d \xi
$$

where

$$
e_{D}=1-\exp \left(-\mathrm{i} \kappa y_{2}\right) \frac{\sin \left(\kappa\left((\tilde{L}+H)-y_{2}\right)\right)}{\sin (\kappa(\tilde{L}+H))}=\exp (2 \mathrm{i} \kappa(\tilde{L}+H)) \frac{\left(\exp \left(-2 \mathrm{i} \kappa y_{2}\right)-1\right)}{(1-\exp (2 \mathrm{i} \kappa(\tilde{L}+H)))} .
$$

In other words,

$$
u(x)-u_{p, d}(x)=\frac{-\mathrm{i}}{\pi} \int_{-\infty}^{\infty} \exp \left(-\mathrm{i} \xi x_{1}\right) \sin \left(\kappa x_{2}\right) \sin \left(\kappa y_{2}\right) \frac{\exp (2 \mathrm{i} \kappa(\tilde{L}+H))}{\kappa(1-\exp (2 \mathrm{i} \kappa(\tilde{L}+H)))} d \xi .
$$

The same expression is obtained in the case $y_{2}<x_{2} \leq H$.

We now make the substitution $\xi=k \sin \theta$, so that $\kappa=k \cos \theta$, where $\theta$ runs over the contour $C$ shown in Fig. 4.1, to obtain

$$
\begin{aligned}
& u(x)-u_{p, d}(x) \\
& =\frac{-\mathrm{i}}{\pi} \int_{C} \exp \left(-\mathrm{i} k x_{1} \sin \theta\right) \sin \left(k x_{2} \cos \theta\right) \sin \left(k y_{2} \cos \theta\right) \frac{\exp (2 \mathrm{i} k(\tilde{L}+H) \cos \theta)}{1-\exp (2 \mathrm{i} k(\tilde{L}+H) \cos \theta)} d \theta .
\end{aligned}
$$


Note that the integrand is analytic except for poles that lie in the shaded regions in Fig. 4.1 (but not on their boundaries). Now, writing $\theta=\theta_{x}+\mathrm{i} \theta_{y}$, we note that as $\theta_{y} \rightarrow+\infty$ with $-\pi / 2 \leq \theta_{x} \leq 0$, $\exp (2 \mathrm{i} k(\tilde{L}+H) \cos \theta) \rightarrow 0$, uniformly in $\theta_{x}$, since $\Re(\tilde{L})>0$ and $\Im(\tilde{L})>0$. Thus, for $-\pi / 2 \leq \theta_{x} \leq 0$ and all $\theta_{y}$ sufficiently large, the modulus of the integrand in $(4.31)$ is $\leq 2 \exp (D(\theta))$, where

$$
D(\theta)=k\left|\sinh \theta_{y}\right|\left[\left(x_{2}+y_{2}-2(\Re(\tilde{L})+H)\right)\left|\sin \theta_{x}\right|+\left(\left|x_{1}\right|-2 \Im(\tilde{L})\right) \cos \theta_{x}\right] .
$$

The same bound holds for $0 \leq \theta_{x} \leq \pi / 2$ if $\theta_{y}$ is sufficiently large and negative. Thus, for $\left|x_{1}\right|<2 \Im(\tilde{L})$, we can deform the contour $C$ to the imaginary axis, i.e. to the contour $\theta=-\mathrm{i} t,-\infty<t<+\infty$, so that

$$
\begin{aligned}
& u(x)-u_{p, d}(x) \\
& =\frac{-1}{\pi} \int_{-\infty}^{\infty} \exp \left(k x_{1} \sinh t\right) \sin \left(k x_{2} \cosh t\right) \sin \left(k y_{2} \cosh t\right) \frac{\exp (2 \mathrm{i} k(\tilde{L}+H) \cosh t)}{1-\exp (2 \mathrm{i} k(\tilde{L}+H) \cosh t)} d t .
\end{aligned}
$$

Now using the substitution $\cosh t=1+u^{2}$ and $\sinh t=u \sqrt{2+u^{2}}$ we obtain

$$
\begin{aligned}
& u(x)-u_{p, d}(x) \\
& =\frac{-2}{\pi} \int_{-\infty}^{\infty} \exp \left(k x_{1} u \sqrt{2+u^{2}}\right) \sin \left(k x_{2}\left(1+u^{2}\right)\right) \sin \left(k y_{2}\left(1+u^{2}\right)\right) \\
& \quad \frac{\exp \left(2 \mathrm{i} k(\tilde{L}+H)\left(1+u^{2}\right)\right)}{1-\exp \left(2 \mathrm{i} k(\tilde{L}+H)\left(1+u^{2}\right)\right)} \frac{d u}{\sqrt{2+u^{2}}} .
\end{aligned}
$$

Thus, using the fact that $\Im(\tilde{L})>0$ and $\Re(\tilde{L})>0$,

$$
\begin{aligned}
\left|u(x)-u_{p, d}(x)\right| & \leq \frac{2}{\pi} \int_{-\infty}^{\infty} \exp \left(k x_{1} u \sqrt{2+u^{2}}\right) \frac{\exp \left(-2 k \Im(\tilde{L})\left(1+u^{2}\right)\right)}{1-\exp (-2 k \Im(\tilde{L}))} \frac{d u}{\sqrt{2}} \\
& \leq \frac{\sqrt{2} \exp (-2 k \Im(\tilde{L})}{\pi(1-\exp (-2 k \Im(\tilde{L})))} \int_{-\infty}^{\infty} \exp \left(k\left(x_{1} u \sqrt{2+u^{2}}-2 \Im(\tilde{L}) u^{2}\right)\right) d u .
\end{aligned}
$$

Noting that this last integral is even as a function of $x_{1}$, assume without loss of generality that $x_{1} \geq 0$. Then the integral may be estimated by

$$
\begin{aligned}
\int_{-\infty}^{\infty} \exp \left(k\left(x_{1} u \sqrt{2+u^{2}}-2 \Im(\tilde{L}) u^{2}\right)\right) d u \leq & \left.\int_{-\infty}^{0} \exp \left(-2 k \Im(\tilde{L}) u^{2}\right)\right) d u \\
& +\int_{0}^{\infty} \exp \left(k\left(x_{1} u(\sqrt{2}+u)-2 \Im(\tilde{L}) u^{2}\right)\right) d u
\end{aligned}
$$

Using the fact that, if $a>0$ and $b>0$,

$$
\int_{0}^{\infty} \exp \left(a u-b u^{2}\right) d u=\sqrt{\pi} \exp \left(a^{2} / 4 b\right) \frac{(\operatorname{erf}(a / 2 \sqrt{b})+1)}{2 \sqrt{b}} \leq \sqrt{\pi} \frac{\exp \left(a^{2} / 4 b\right)}{\sqrt{b}} \text { and } \int_{-\infty}^{0} \exp \left(-a u^{2}\right) d u=\frac{\sqrt{\pi}}{2 \sqrt{a}},
$$

we obtain the estimate (4.26).

For the case of a Neumann boundary condition we now consider $u-u_{p, n}$ and find that the analogue of (4.30) holds with $e_{D}$ replaced by

$$
e_{N}=1-\exp \left(-\mathrm{i} \kappa y_{2}\right) \frac{\cos \left(\kappa\left(y_{2}-(\tilde{L}+H)\right)\right)}{\cos (\kappa(\tilde{L}+H))}=\exp (2 \mathrm{i} \kappa(\tilde{L}+H)) \frac{1-\exp \left(-2 \mathrm{i} \kappa y_{2}\right)}{1+\exp (2 \mathrm{i} \kappa(\tilde{L}+H))} .
$$

Deforming the contour and using the same substitution as above we find the following equality:

$$
\begin{aligned}
& u(x)-u_{p, n}(x) \\
& =\frac{2}{\pi} \int_{-\infty}^{\infty} \exp \left(k x_{1} u \sqrt{2+u^{2}}\right) \sin \left(k x_{2}\left(1+u^{2}\right)\right) \sin \left(k y_{2}\left(1+u^{2}\right)\right) \\
& \quad \frac{\exp \left(2 \mathrm{i} k(\tilde{L}+H)\left(1+u^{2}\right)\right)}{\exp \left(2 \mathrm{i} k(\tilde{L}+H)\left(1+u^{2}\right)\right)+1} \frac{d u}{\sqrt{2+u^{2}}} .
\end{aligned}
$$



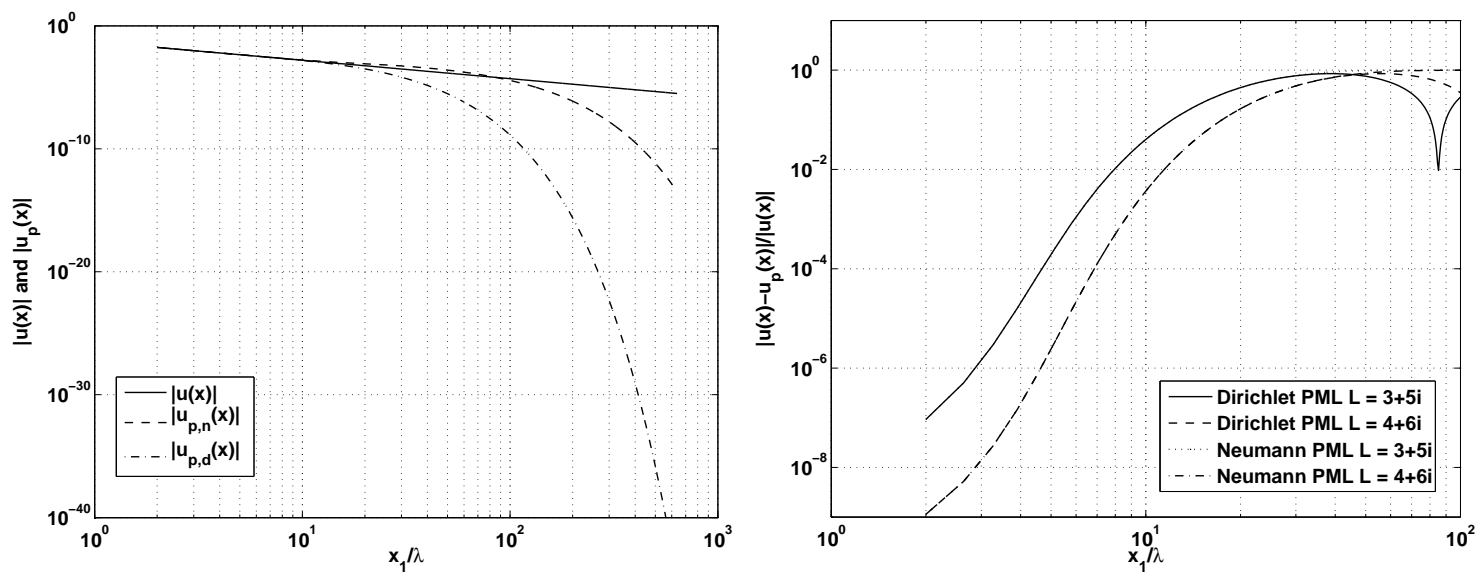

Fig. 4.2. A computational study of the convergence of $u_{p, d}$ and $u_{p, n}$ to $u$ for a flat surface. In the left panel we show that the PML solution is damped exponentially as $x_{1}$ increases, for both a Dirichlet and Neumann backing to the PML. In the right hand panel we show that excellent relative error, with a rapid convergence rate, can be obtained close to $x_{1}=0$ but that the solution deteriorates further away. For the parameters behind these graphs, see the text (note that the label $L$ in the right panel refers to $\tilde{L} !)$.

The estimate of the right hand side proceeds as before and completes the proof of the theorem.

口

We end this section with some numerical results that illustrate our theoretical results for a particular choice of parameters. Choosing arbitrarily $k=2, H=2, x_{2}=1$ and $y_{2}=0.5$, we can evaluate the exact solution $u(x)$ given by (4.1). We can also add the first $N$ terms of (4.6) and (4.7) to obtain accurate estimates of $u_{p, d}(x)$ and $u_{p, n}(x)$ when we choose $\tilde{L}=3+5 \mathrm{i}$ (after some experimentation we use $N=100$ ).

In Fig. 4.2 (left panel) we show a $\log$-log plot of $|u(x)|,\left|u_{p, d}(x)\right|$ and $\left|u_{p, n}(x)\right|$ as a function of $x_{1} / \lambda$, where $\lambda=2 \pi / k$ is the wavelength. The figure shows the expected rate of decay of $O\left(\left|x_{1}\right|^{-3 / 2}\right)$ for $u$, whereas $u_{p, d}$ and $u_{p, n}$ both ultimately decay exponentially as we predict in Lemma 4.1. In line with the predictions in this lemma the exponential decay of the Dirichlet PML solution is much faster (compare (4.12) with (4.9)). With this choice of parameters, the PML and exact solutions do not differ in norm appreciably until approximately 10 wavelengths from the source; thereafter $\left|u_{p, d}(x)\right|$ diverges from $|u(x)|$ more rapidly.

In Fig. 4.2 (right panel) we show a log-log plot of the relative error $\left|u(x)-u_{p, d}(x)\right| /|u(x)|$ as a function of the normalized position $x_{1} / \lambda$ (both for our previous choice of $\tilde{L}$ and for the stronger layer $\tilde{L}=4+6 \mathrm{i}$ ). As is to be expected, close to $x_{1}=0$ the PML solution is very accurate. Indeed the relative error is better than $10^{-4 \%}$ in both cases at $x_{1}=0$, but at 10 wavelengths from the source, the error has increased to $4 \%$ when $\tilde{L}=3+5 \mathrm{i}$. The rapid convergence of the solution at $x_{1}=0$ is also clear from this figure. Surprisingly, perhaps, in view of the left hand graph, the relative errors in $u_{p, d}(x)$ and $u_{p, n}(x)$ appear identical up to $x_{1} / \lambda \approx 30$ for $\tilde{L}=3+5 \mathrm{i}$, up to $x_{1} / \lambda \approx 50$ for $\tilde{L}=4+6 \mathrm{i}$.

5. An iterative scheme. In Section 2 we showed that the PML solution $u_{p}$ converges to the true solution $u$ of the rough surface scattering problem as the virtual layer thickness increases. However the results suggest that the convergence rate may be as slow as first order.

We now propose to use the PML as part of an iterative scheme for correcting the PML solution $u_{p}$. The method is based on the overlapping domain decomposition methods of [13] and the iterative scheme of [18]. However, unlike [18], we use the PML so that convergence can be proved.

We assume that the layer has been chosen sufficiently thick that the PML problem has a unique solution (see Theorem 3.3) with a positive inf-sup constant $\gamma_{p}$. Specifically, we assume that $2 C_{U}(\alpha, \beta)<\gamma$ so that, from (3.3),

$$
\gamma_{p} \geq \gamma-2 C_{U}(\alpha, \beta)>0
$$

We first consider the following PML problem with non-homogeneous Neumann data on $\Gamma_{H+L}$. Suppose 
$f \in H^{-1 / 2}\left(\Gamma_{H+L}\right)$ is a given function. We want to study the problem of finding $v \in V_{H+L}$ such that

$$
\begin{aligned}
\frac{1}{\sigma} \frac{\partial v}{\partial x_{n}} & =f \text { on } \Gamma_{H+L}, \\
\Delta_{p} v+k^{2} \sigma v & =g \text { in } S_{H+L} .
\end{aligned}
$$

Proceeding as for the simple PML, we see that $v \in V_{H}$ satisfies

$$
-\int_{S_{H}} g \bar{\phi} d x=\int_{S_{H}} \nabla v \cdot \nabla \bar{\phi}-k^{2} v \bar{\phi} d x-\int_{\Gamma_{H}} \bar{\phi} \frac{\partial v}{\partial x_{n}} d s
$$

for all $\phi \in V_{H}$ and using the PML approximation to the Dirichlet-to-Neumann map (2.12) we have

$$
-\left.\frac{\partial v}{\partial x_{n}}\right|_{x_{n}=H}=\left.T_{p} v\right|_{x_{n}=H}+N_{p} f
$$

Thus the restriction of the solution $v$ of (5.2)-(5.3) to $S_{H}$ (still denoted $v$ !) is characterized variationally by requiring that $v \in V_{H}$ satisfy

$$
b_{p}(v, \phi)=-\int_{\Gamma_{H}} \bar{\phi} N_{p} f d s+\int_{S_{H}} g \bar{\phi} d x \text { for all } \phi \in V_{H} .
$$

The Babuška-Brezzi theory implies that this problem has a unique solution for any $f \in H^{-1 / 2}\left(\Gamma_{H+L}\right)$ (see the proof of the invertibility of $B_{p}$ in Section 3) since $N_{p}$ is a bounded operator from $H^{-1 / 2}\left(\Gamma_{H+L}\right)$ to $H^{-1 / 2}\left(\Gamma_{H}\right)$.

We can also consider the use of an infinite PML using $\sigma=\sigma(H+L)$ to extend the given PML parameter $\sigma$ to infinity. Our convergence result in Theorem 3.3 implies that the resulting field, denoted $u_{p, \infty}$ agrees with $u$ on $S_{H}$ and has the representation, in Fourier space, as follows

$$
\hat{u}_{p, \infty}\left(\xi, x_{n}\right)=\exp \left(-z\left(\hat{x}_{n}-H\right)\right) \hat{u}_{p, \infty}(\xi, H) \text { for } x_{n}>H .
$$

Thus the infinite PML gives rise to Neumann data on $\Gamma_{H+L}$ given, in the Fourier domain, by

$$
\left.\frac{1}{\sigma} \frac{\partial \hat{u}_{p, \infty}}{\partial x_{n}}\right|_{x_{n}=H+L}=-z(\xi) \exp (-z(\xi) \tilde{L}) \hat{u}_{p, \infty}(\xi, H) .
$$

We can then define an extension operator $E_{p}: H^{1 / 2}\left(\Gamma_{H}\right) \rightarrow H^{-1 / 2}\left(\Gamma_{H+L}\right)$ such that if $\eta \in H^{1 / 2}\left(\Gamma_{H}\right)$ then

$$
\widehat{E_{p} \eta}=-z \exp (-z \tilde{L}) \hat{\eta} .
$$

We see that the infinite PML solution $u_{p, \infty} \in V_{H}$ satisfies

$$
b_{p}\left(u_{p, \infty}, \phi\right)=\int_{\Gamma_{H}} \bar{\phi} N_{p} E_{p} u_{p, \infty} d s+\int_{S_{H}} g \bar{\phi} d x \text { for all } \phi \in V_{H},
$$

and, since $u_{p, \infty}=u$ on $S_{H}$,

$$
b_{p}(u, \phi)=\int_{\Gamma_{H}} \bar{\phi} N_{p} E_{p} u d s+\int_{S_{H}} g \bar{\phi} d x \text { for all } \phi \in V_{H} .
$$

The finite element discretization of this problem (including the PML layer) would give a method akin to that of [13] but using a PML in place of a free space layer on the strip $S_{H}^{L}$. The advantage of the PML is that it can be used to provide a provably convergent iterative method. In particular we propose a method like that of [18] as follows. Given an initial guess $u^{(0)} \in V_{H}$ (for example $u^{(0)}=0$ ) we define $u^{(n)} \in V_{H}$ for $n=1,2, \ldots$ by requiring that

$$
b_{p}\left(u^{(n)}, \phi\right)=\int_{\Gamma_{H}} \bar{\phi} N_{p} E_{p} u^{(n-1)} d s+\int_{S_{H}} g \bar{\phi} d x \text { for all } \phi \in V_{H} .
$$


As argued earlier in this section, if $\tilde{L}$ is chosen appropriately we can ensure that the above problem has a unique solution for each $n$ and so the iteration is well defined. Of course in practice we shall use a finite element approximation of the boundary value problem of finding $u^{(n)} \in V_{H+L}$ such that

$$
\begin{aligned}
\frac{1}{\sigma} \frac{\partial u^{(n)}}{\partial x_{n}} & =E_{p} u^{(n-1)} \text { on } \Gamma_{H+L}, \\
\Delta_{p} u^{(n)}+k^{2} \sigma u^{(n)} & =g \text { in } S_{H+L} .
\end{aligned}
$$

Thus at each iteration we must evaluate $E_{p}$ (using the Fast Fourier Transform) and then solve a finite element problem on the strip $S_{H+L}$ (in practice, truncated laterally). More details of one possible finite element method are given in the next section.

We now want to investigate the convergence of the scheme. Using the PML inf-sup condition and the trace estimate (3.1),

$$
\begin{aligned}
\gamma_{p}\left\|u-u^{(n)}\right\|_{V} & \leq \sup _{\phi \in V_{H}} \frac{\left|b\left(u-u^{(n)}, \phi\right)\right|}{\|\phi\|_{V_{H}}} \\
& =\sup _{\phi \in V_{H}} \frac{\left|\int_{\Gamma_{H}} \bar{\phi} N_{p} E_{p}\left(u-u^{(n-1)}\right) d s\right|}{\|\phi\|_{V_{H}}} \\
& \leq 2\left\|N_{p} E_{p}\right\|_{\mathcal{L}\left(H^{1 / 2}\left(\Gamma_{H}\right), H^{-1 / 2}\left(\Gamma_{H}\right)\right.}\left\|u-u^{(n-1)}\right\|_{V_{H}} .
\end{aligned}
$$

It remains to estimate $\left\|N_{p} E_{p}\right\|_{\mathcal{L}\left(H^{1 / 2}\left(\Gamma_{H}\right), H^{-1 / 2}\left(\Gamma_{H}\right)\right.}$. From the Fourier representation of $N_{p}$ and $E_{p}$ we see that, in the Fourier domain, the action of $N_{p} E_{p}$ corresponds to multiplication by

$$
z_{N E}=\frac{2 z \exp (-z \tilde{L})}{\exp (z \tilde{L})+\exp (-z \tilde{L})} .
$$

To estimate the operator norm it therefore suffices to bound

$$
\max _{\xi \in R} \frac{\left|z_{N E}(\xi)\right|}{\sqrt{k^{2}+\xi^{2}}}
$$

But this has already been done in Theorem 3.1 and we conclude that

$$
\left\|N_{p} E_{p}\right\|_{\mathcal{L}\left(H^{1 / 2}\left(\Gamma_{H}\right), H^{-1 / 2}\left(\Gamma_{H}\right)\right.} \leq C_{U}(\alpha, \beta) .
$$

Using the estimate (5.1) for $\gamma_{p}$ this implies that

$$
\left\|u-u^{(n)}\right\|_{V_{H}} \leq \frac{2 C_{U}(\alpha, \beta)}{\gamma-2 C_{U}(\alpha, \beta)}\left\|u-u^{(n-1)}\right\|_{V_{H}} .
$$

The constant $2 C_{U}(\alpha, \beta) /\left(\gamma-2 C_{U}(\alpha, \beta)\right)$ can be made less than one by choosing $\alpha$ and $\beta$ large enough, while retaining some constraint on the ratio $\alpha / \beta$, and in that case the iterative scheme will converge. Note that this is less restrictive than having to choose $\alpha$ and $\beta$ so that

$$
2 C_{U}(\alpha, \beta) /\left(\gamma-2 C_{U}(\alpha, \beta)\right)<\epsilon
$$

which is required to ensure a relative error $\epsilon$ by our convergence result in Theorem 3.3. Thus the PML can be thinner. The price to be paid for the thinner PML is that at each iteration we must compute the action of the operator $E_{p}$. But this is not a singular integral operator and the action can be computed efficiently via the Fast Fourier Transform as we shall see in the next section.

In summary we have proved the following theorem.

ThEOREM 5.1. Suppose D satisfies the boundary constraint (1.8). If $\sigma$ is chosen so that $4 C_{U}(\alpha, \beta)<\gamma$ then the iterative scheme defined by (5.4) is well defined and $u^{(n)}$ converges linearly to the exact solution $u$ according to (5.7). 
6. Numerical results. So far we have assumed that the data $g$ is supported in $S_{H}$. This can be inconvenient since we want to take $H$ as small as possible in order to decrease the thickness of the region to be covered by finite elements (or we may wish to use a point source that is not in $V_{H}^{*}$ ). To handle this case we define the incident field denoted $u_{h}^{I}$ by

$$
u_{h}^{I}(x)=-\int_{D} \mathcal{G}_{h}(x, y) g(y) d y \text { for } x \in U_{h},
$$

where $\mathcal{G}_{h}$ is the Dirichlet Green's function for the half-space $U_{h}$ above $\Gamma_{h}$ for some $h<f_{-}$. In this case

$$
\mathcal{G}_{h}(x, y)=\Phi(x, y)-\Phi\left(x, y_{h}^{\prime}\right)
$$

where, if $y=\left(\tilde{y}, y_{n}\right)$, the reflected point $y_{h}^{\prime}=\left(\tilde{y}, 2 h-y_{n}\right)$, and $\Phi(x, y):=\frac{\mathrm{i}}{4} H_{0}^{(1)}(k|x-y|)$ in $2 \mathrm{D},:=$ $\exp (\mathrm{i} k|x-y|) /(4 \pi|x-y|)$ in $3 \mathrm{D}$, is the standard fundamental solution of the Helmholtz equation. Restricting attention to the case when $\Gamma$ is Lipschitz, specifically the graph of a bounded and uniformly Lipschitz function, in which case there exists a well-defined trace operator from $H^{1}(D)$ to $H^{1 / 2}(\Gamma)$, we can allow in the theory any incident field such that $u_{h}^{I} \in H^{1 / 2}(\Gamma)$, so we can also allow the point source $g=-\delta_{z}\left(\delta_{z}\right.$ is the delta distribution concentrated at $\left.z=\left(z_{1}, z_{2}\right)\right)$ provided $z_{2}>f\left(z_{1}\right)$. In this case

$$
u_{h}^{I}(x)=\mathcal{G}_{h}(x, z) .
$$

Our computational results will all be for the point source.

Using the incident field $u_{h}^{I}$, we define the scattered field $u^{s}$ by $u^{s}=u-u_{h}^{I}$. Then $u^{s} \in H^{1}\left(S_{H}\right)$ for every $H \geq f_{+}$satisfies

$$
\begin{aligned}
u^{s} & =-u_{h}^{I} \text { on } \Gamma, \\
\Delta u^{s}+k^{2} u^{s} & =0 \text { in } D,
\end{aligned}
$$

together with the radiation condition (1.4). Using (2.3) this problem is equivalent to the variational problem of finding the function $w \in H^{1}\left(S_{H}\right)$ such that

$$
\begin{aligned}
u^{s} & =-u_{h}^{I} \text { on } \Gamma, \\
b\left(u^{s}, \phi\right) & =0 \text { for all } \phi \in V_{H} .
\end{aligned}
$$

Let us first consider the simple PML problem (2.9)-(2.10). In practice we do not use the operators $T_{p}$. Instead the computational domain is $S_{H+L}=S_{H} \cup S_{H}^{L} \cup \Gamma_{H}$. Defining

$$
A=\left(\begin{array}{cc}
\sigma & 0 \\
0 & (1 / \sigma)
\end{array}\right)
$$

we see that $u_{p}^{s}$ satisfies

$$
\begin{aligned}
u_{p}^{s} & =-u_{h}^{I} \text { on } \Gamma_{H}, \\
\nabla \cdot\left(A \nabla u_{p}^{s}\right)+k^{2} \sigma u_{p}^{s} & =0 \text { in } S_{H+L}, \\
\frac{1}{\sigma} \frac{\partial u_{p}^{s}}{\partial x_{n}} & =0 \text { on } \Gamma_{H+L} .
\end{aligned}
$$

This can be formulated variationally by requiring that $u_{p}^{s} \in H^{1}\left(S_{H+L}\right)$ together with (6.5) and

$$
a\left(u_{p}^{s}, \phi\right)=0 \text { for all } \phi \in V_{H+L}
$$

where

$$
a\left(u_{p}^{s}, \phi\right)=\int_{S_{H+L}}\left(A \nabla u_{p}^{s} \cdot \nabla \bar{\phi}-k^{2} \sigma u_{p}^{s} \bar{\phi}\right) d x .
$$

This problem can then be discretized by finite element methods. In this paper we shall not analyze the finite element problem. We use cubic finite elements on a triangular mesh, with the boundary data being interpolated at the Gauss-Lobatto points on each edge of the mesh on $\Gamma$. 


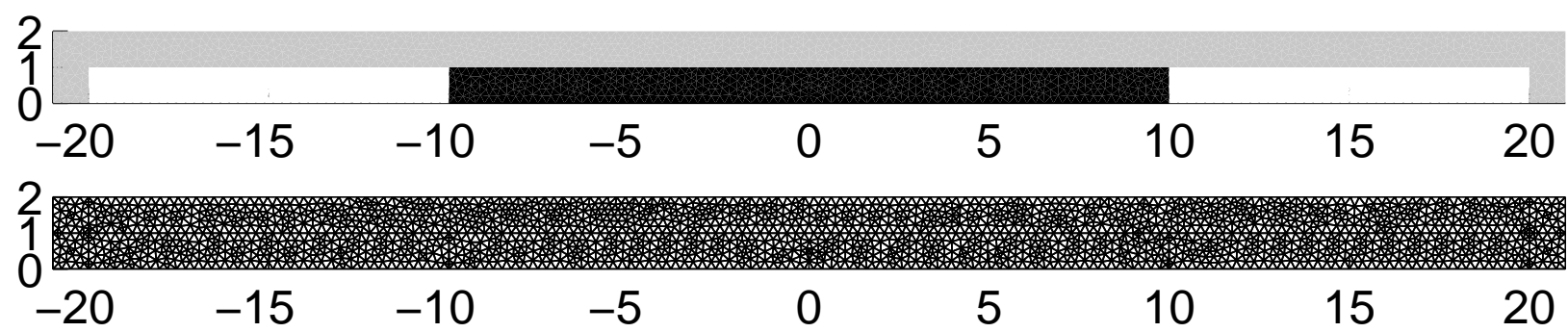

FIG. 6.1. The top panel shows the domain of the computation. The gray region is PML. The black and white regions are the truncated $S_{H}$ and the black region is where the error between the exact and PML/FEM solution is computed. The lower figure shows the mesh which is very fine since we wish to study effects of the PML rather than the mesh.

6.1. A flat scatterer. Here we provide some numerical tests of the straightfoward use of the PML to terminate the model problem of computing the solution of scattering of the field due to a point source above an infinite flat boundary. Of course this is a special case, but it has the advantage that we know the exact solution.

In this case $D=U_{0}$ and $\Gamma=\left\{\left(x_{1}, 0\right): x_{1} \in \mathbb{R}\right\}$. The point source is located at $y=\left(0, y_{2}\right), y_{2}>0$. Using the image principle it is then immediate that the total field in $U_{0}$ is

$$
u(x)=\Phi(x, y)-\Phi\left(x, y^{\prime}\right)
$$

where $y^{\prime}=\left(0,-y_{2}\right)$ is the image point.

We choose as an incident field

$$
u^{i}(x)=\Phi(x, y)-\Phi\left(x, y_{h}^{\prime}\right)
$$

where $y_{h}^{\prime}=\left(0,2 h-y_{2}\right)$ and $h<0$ is a parameter and $u^{i}$ is analytic in a neighborhood of $\Gamma$. This incident field has the same decay as the solution as $\left|x_{1}\right| \rightarrow \infty$. The exact scattered field is

$$
u^{s}(x)=u(x)-u^{i}(x)=\Phi\left(x, y_{h}^{\prime}\right)-\Phi\left(x, y^{\prime}\right) .
$$

The computational domain is truncated laterally at $x_{1}=-A$ and $x_{1}=A$ using a PML of width $L$ in the $\pm x_{1}$ directions. This aspect of the truncation procedure is not captured by our preceding analysis. For a simple model problem we choose the parameter values given in the following table. The PML parameter $\sigma$ is given by (2.8) in $\left|x_{1}\right| \leq A$ and by the same formula with $x_{2}$ replaced by $x_{1}$ and $H$ replaced by $A$ on the ends $A \leq\left|x_{1}\right| \leq A+L$.

\begin{tabular}{|cc||cc|}
\hline Parameter & Value & Parameter & Value \\
\hline$H$ & 1 & $L$ & 1 \\
$m$ & 1 & $\sigma_{0}$ & variable \\
$A$ & 20 & $k$ & 4 \\
$y_{2}$ & 3 & $h$ & -1 \\
\hline
\end{tabular}

The mesh has a maximum element diameter of 0.32 and a diagram of the domain and the mesh is shown in Figure 6.1.

Results are shown in Figure 6.2. As might be expected from Theorem 4.6 and particularly from (4.29) in Remark 4.7, we see exponential convergence in $\Im(\tilde{L})$ and little effect of increasing $\Re(\tilde{L})$.

As in most of the rest of this paper, we have chosen to use the Neumann boundary condition on the PML which slightly simplifies the numerical analysis compared to a Dirichlet condition. To test if this choice has an adverse effect on accuracy we next compare results for the Dirichlet and Neumann boundary condition for the problem just considered. The error graph for the Dirichlet case is shown in Figure 6.3. As expected from the analytic results in Section 4 there is little difference between the results in Figures 6.2 and 6.3 for the two boundary conditions. 


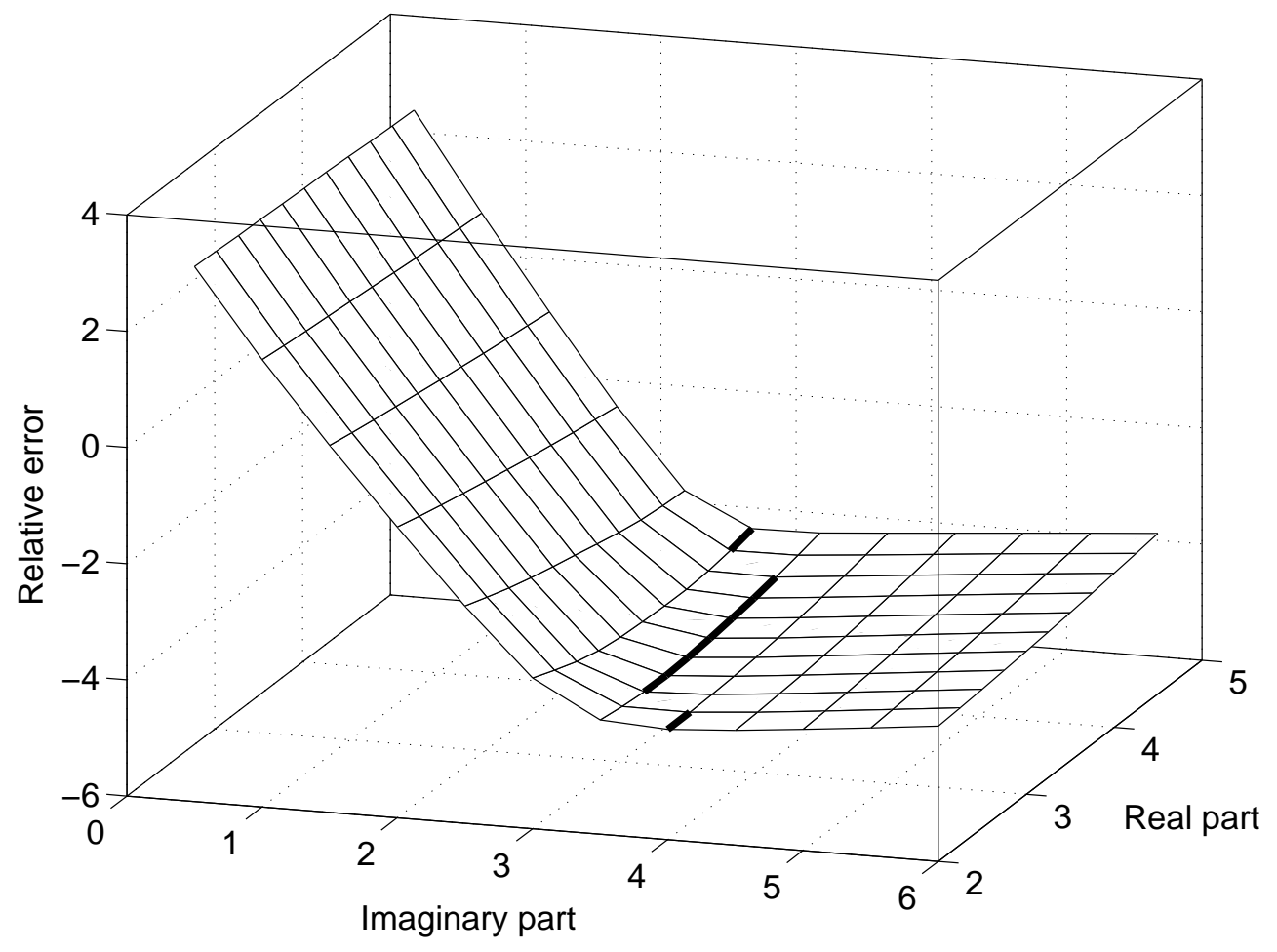

FIG. 6.2. A surface plot of the base 10 logarithm of the discrete relative $L^{2}$ error for the PML/finite element solution against the real and imaginary parts of $\tilde{L}$ (using a Neumann boundary condition on the PML). The solid line marks the minimum error for fixed real part as the imaginary part is varied. Clearly this graph suggests exponential convergence as the imaginary part of $\sigma$ increases and almost no dependence on the real part.

6.2. The iterative scheme. Next we shall test the convergence of the iterative scheme described in Section 5. The problem is exactly the same as for the Neumann-PML problem in the previous section (a flat surface) and the parameters are identical except that we now fix $\sigma_{0}=1 / 2$ (corresponding to $\Im(\tilde{L})=1$ ). From the results shown in Figure 6.2 we know that this choice of $\sigma_{0}$ is too small and will result in a significant error (in fact $25 \%$ in the relative discrete $L^{2}\left(S_{H}\right)$ norm in the subdomain used for Fig. 6.2). Applying the iterative correction algorithm gives the results shown in Table 6.1. The iterative scheme greatly improves the solution, but the error stagnates at about $4 \%$ (higher than can be achieved by an optimal choice of the PML parameter - see Fig. 6.2). There are multiple factors contributing to this error:

- The computation of $E_{p}$ using the Fast Fourier Transform provides an approximation to the exact $E_{p}$, and $E_{p}$ is not the finite element extension operator.

- Our analysis does not include the effects of the lateral PML. In our code this uses the same absorption parameter $\sigma_{0}$ as the vertical PML, which is deliberately chosen too small. Thus lateral absorption is poor and not corrected by the use of the iterative scheme

Our results do show that the iterative scheme can improve the solution even if the PML parameter is not chosen optimally.

7. Conclusion. In this paper we have investigated the use of the PML to truncate a rough surface scattering problem in the direction away from the scatterer. We have provided a worst case estimate showing only linear convergence of the solution in the global $V_{H}$ norm as the layer is made thicker. We have also shown, by more detailed study of one special case (a point source above a flat scatterer), that this estimate of the rate of convergence in the global $V_{H}$ norm is fairly sharp, in particular there is no exponential convergence globally, because the PML causes the solution to decay exponentially at infinity. Nevertheless, we have also proved in this special case, and see in the finite element simulations, exponential convergence on compact subsets. In view of this wide range of possible behaviors, and because the PML parameter is difficult to 


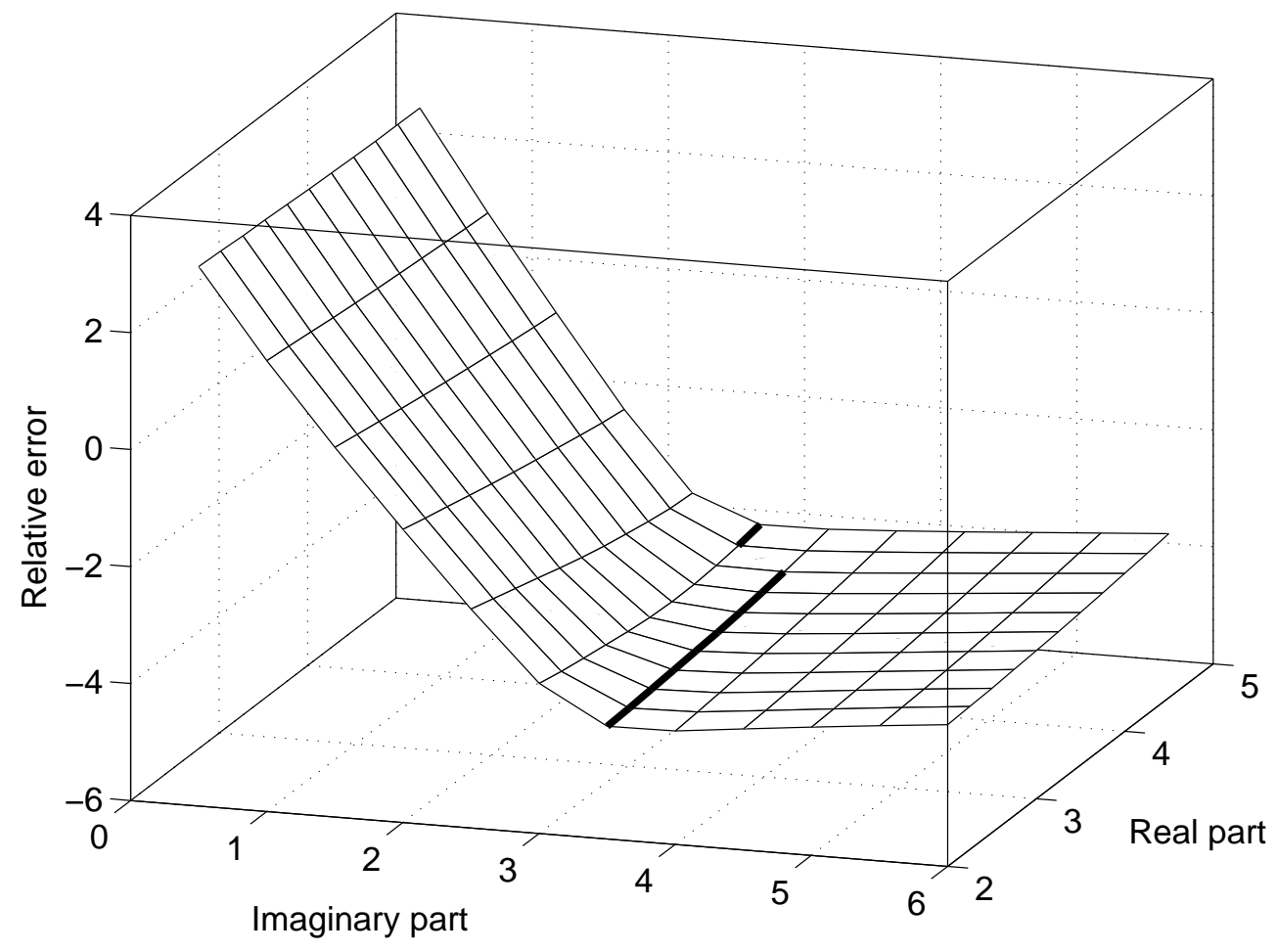

FIG. 6.3. A surface plot of the base 10 logarithm of the discrete relative $L^{2}$ error for the PML/finite element solution against the real and imaginary parts of $\tilde{L}$ using the homogeneous Dirichlet boundary condition on the PML. Generally the results are similar to those computed using the Neumann boundary condition in Fig. 6.2.

\begin{tabular}{||l|l||}
\hline \hline Iteration number & Relative discrete error \\
\hline 1 & $24.8 \%$ \\
2 & $5.54 \%$ \\
3 & $3.69 \%$ \\
4 & $4.33 \%$ \\
\hline \hline
\end{tabular}

TABLE 6.1

Error in successive iterates of the iterative correction scheme. After $n=4$ the error stagnates at about $4 \%$

pick a priori, we have also proposed a simple iterative scheme to correct the PML solution, proving linear convergence, which we illustrate with numerical results for a simple case.

Three important questions are unanswered by this study:

1. How is the method influenced by lateral termination?

2. What is the error for the finite element method applied to the truncated problem (the difficulty is to obtain the dependence of the error on the PML parameters and lateral cutoff)?

3. In the general case, is convergence exponential on compact subsets of $S_{H}$ ?

Finally we have not addressed the practical problem of how to solve the linear system resulting from the PML-variational finite element scheme (in this study, in $\mathbb{R}^{2}$, direct solution is used). We hope to make progress on these questions in the future.

\section{REFERENCES}

[1] M. Abramowitz And I. A. Stegun, Handbook of Mathematical Functions, Dover, New York, 1972. 
[2] T. Arens and T. Hohage, On radiation conditions for rough surface scattering problems, IMA J. Applied Math., 70 (2005), pp. 839-847.

[3] G. BaO, D. Dobson, And J. Cox, Mathematical studies in rigorous grating theory, J. Opt. Soc. Amer. A, 12 (1995), pp. 1029-1042.

[4] S. Chandler-Wilde And P. Monk, Existence, uniqueness and variational methods for scattering by unbounded rough surfaces, SIAM J. Math. Anal., 37 (2005), pp. 598-618.

[5] S. Chandler-Wilde, P. Monk, And M. Thomas, The mathematics of scattering by unbounded, rough, inhomogeneous layers, J. Comp. Appl. Math., 204 (2007), pp. 549-559 .

[6] S. Chandler-Wilde, M. Rahman, and C. Ross, A fast two-grid and finite section method for a class of integral equations on the real line with application to an acoustic scattering problem in the half-plane, Numer. Math., 93 (2002), pp. 1-51.

[7] Z. Chen AND H. Wu, An adaptive finite element method with perfectly matched absorbing layers for the wave scattering by periodic structures, SIAM J. Numer. Anal., 41 (2003), pp. 799-826.

[8] W. C. Chew and W. H. Weedon, A 3D perfectly matched medium from modified Maxwell's equations with stretched coordinates, Microwave Opt. Technol. Lett., 7 (1994), pp. 599-604.

[9] F. Collino And P. Monk, The perfectly matched layer in curvilinear coordinates, SIAM J. Sci. Comput., 19 (1998), pp. 2061-90.

[10] J. A. DeSanto, Scattering by rough surfaces, in Scattering: Scattering and Inverse Scattering in Pure and Applied Science, R. Pike and P. Sabatier, eds., Academic Press, 2002, pp. 15-36.

[11] J. Diaz And P. Joly, A time domain analysis of PML models in acoustics, Comp. Meth. Appl. Mech. Eng. 195 (2006), pp. 3820-3853.

[12] J. Elschner and G. Schmidt, Diffraction in periodic structures and optimal design of binary gratings I: Direct problems and gradient formulas, Math. Methods Appl. Sci., 81 (1998), pp. 1307-1328.

[13] A. Jami And M. Lenoir, A variational formulation for exterior problems in linear hydrodynamics, Comput. Meth. Appl. Mech. Eng., 16 (1978), pp. 341-59.

[14] R. Kress, Linear Integral Equations, Springer, Berlin, 2nd ed., 1999.

[15] M. Lassas and E. Somersalo, Analysis of the PML equations in general convex geometry, Proc. Royal Soc. Edinburgh, 131 (2001), pp. 1183-207.

[16] M. Lassas And E. Sommersalo, On the existence and convergence of the solution of pml equations, Computing, 60 (1998), pp. 229-42.

[17] C.M. Linton, The Green's function for the two-dimensional Helmholtz equation in periodic domains, J. Eng. Math., 33 (1998), pp. 377-402.

[18] J. LiU AND J. Jin, A novel hybridization of higher order finite element and boundary integral methods for electromagnetic scattering and radiation problems, IEEE Trans. Antennas Propagat., 49 (2001), pp. 1794-806.

[19] J. A. OGilvy, Theory of Wave Scattering from Random Rough Surfaces, Adam Hilger, Bristol, 1991.

[20] M. Saillard And A. Sentenac, Rigorous solutions for electromagnetic scattering from rough surfaces, Waves Random Media, 11 (2001), pp. R103-R137.

[21] M. Thomas, Analysis of rough surface scattering problems, PhD, University of Reading, 2006.

[22] A. G. Voronovich, Wave Scattering from Rough Surfaces, Springer, Berlin, second ed., 1998.

[23] K. Warnick AND W. C. Chew, Numerical simulation methods for rough surface scattering, Waves Random Media, 11 (2001), pp. R1-R30. 